The Astrophysical Journal, 669:1011-1023, 2007 November 10

(C) 2007. The American Astronomical Society. All rights reserved. Printed in U.S.A.

\title{
CHEMICAL ABUNDANCES OF LUMINOUS COOL STARS IN THE GALACTIC CENTER FROM HIGH-RESOLUTION INFRARED SPECTROSCOPY
}

\author{
Katia CUNha ${ }^{1}$ \\ National Optical Astronomy Observatory, Casilla 603, La Serena, Chile; kcunha@noao.edu \\ Kris Sellgren \\ Department of Astronomy, The Ohio State University, 140 West 18th Avenue, Columbus, OH 43210; sellgren@astronomy.ohio-state.edu \\ Verne V. SMith \\ National Optical Astronomy Observatory, Casilla 603, La Serena, Chile; vsmith@noao.edu \\ SOLANGE V. RAMiREZ \\ Infrared Processing and Analysis Center, California Institute of Technology, Mail Code 100-22, Pasadena, CA 91125; solange@ipac.caltech.edu \\ Robert D. Blum \\ National Optical Astronomy Observatory, P.O. Box 26732, Tucson, AZ 85726; rblum@noao.edu \\ AND \\ DONALD M. TERNDRUP \\ Department of Astronomy, The Ohio State University, 140 West 18th Avenue, Columbus, OH 43210; terndrup@astronomy.ohio-state.edu \\ Received 2007 May 11; accepted 2007 July 16

\begin{abstract}
We present chemical abundances in a sample of luminous cool stars located within $30 \mathrm{pc}$ of the Galactic center. Abundances of carbon, nitrogen, oxygen, calcium, and iron were derived from high-resolution infrared spectra in the $H$ and $K$ bands. The abundance results indicate that both $[\mathrm{O} / \mathrm{Fe}]$ and $[\mathrm{Ca} / \mathrm{Fe}]$ are enhanced, respectively, by averages stars show a nearly uniform and nearly solar iron abundance. The mean value of $A(\mathrm{Fe})=7.59 \pm 0.06$ agrees well with previous work. The total range in Fe abundance among Galactic center stars, 0.16 dex, is significantly narrower than Fe abundance within $30 \mathrm{pc}$ of the Galactic center samples stars with an age less than $1 \mathrm{Gyr}$; a larger sample in time (or space) may find a wider spread in abundances.
\end{abstract} \\ of +0.2 and +0.3 dex, relative to either the Sun or the Milky Way disk at near-solar Fe abundances. The Galactic center \\ the iron abundance distributions found in the literature for the older bulge population. Our snapshot of the current-day
}

Subject headings: Galaxy: center — nuclear reactions, nucleosynthesis, abundances — stars: abundances

\section{INTRODUCTION}

Stellar abundances can provide critical information in understanding the formation and chemical evolution of the halo, disk, and bulge. With the advent of infrared imaging and spectroscopy on large telescopes, it is now possible to explore the stellar population residing near the Galactic center (within $\sim 200 \mathrm{pc}$ ) in detail. Infrared techniques are essential, because this region is obscured by at least $A_{V}=30 \mathrm{mag}$. The Galactic center contains many luminous, massive stars. Those known to date are concentrated in three separate clusters within the central $60 \mathrm{pc}$, the Central Cluster, the Arches Cluster, and the Quintuplet Cluster, at (projected) galactocentric radii $R_{g}$ of 0,24 , and $30 \mathrm{pc}$, respectively. We adopt a distance to the Galactic center of $8.0 \mathrm{kpc}$ (Reid 1993). The youngest stars in these three clusters have ages of 3-9 Myr (Najarro et al. 1994; Krabbe et al. 1995; Blum et al. 1996a), 1-4.5 Myr, and 3-5 Myr (Figer et al. 1998, 1999a, 1999b; Blum et al. 2001), respectively.

The Central Cluster, which defines the dynamical center of the nucleus, has a central stellar density of $4 \times 10^{6} M_{\odot} \mathrm{pc}^{-3}$ at a radius of $\sim 0.3 \mathrm{pc}$ (Ghez et al. 1998; Genzel et al. 2000). The center is believed to contain a massive black hole of (3-4) $\times 10^{6} M_{\odot}$ (Ghez et al. 2005; Schödel et al. 2003). Blum et al. (1996b) have determined $J, H, K$, and $L$ photometry for the 1100 brightest stars in the

\footnotetext{
${ }^{1}$ On leave from Observatório Nacional, Rio de Janeiro, Brazil.
}

Central Cluster (within $5 \mathrm{pc}$ ). Using the observed $J-H, H-K$, and $K-L$ colors and assumed intrinsic colors, they determined the interstellar extinction at $2.2 \mu \mathrm{m}$, finding a mean value of $3.3 \mathrm{mag}$, similar to previous results. The dereddened $K$-band luminosity function of the Central Cluster (Blum et al. 1996b) points to a striking excess of luminous stars compared to the bulge stellar population in Baade's window. These luminous, young (a few Myr old) stars co-exist in the Central Cluster with older stars (Blum et al. 1996a, 2003; Blommaert et al. 1998). Thus, the Galactic center stellar population differs markedly from that in the bulge of the Milky Way, which is mostly made up of stars with ages $\sim 10 \mathrm{Gyr}$, with few stars younger than $5 \mathrm{Gyr}$ (Kuijken \& Rich 2002; Zoccali et al. 2003).

Earlier abundance analysis of the Galactic center include the pioneering studies by Carr et al. (2000) and Ramirez et al. (2000), who analyzed high-resolution $(R=40,000)$ infrared spectra. Carr et al. (2000) derived abundances for the Galactic center supergiant IRS 7, finding nearly solar iron abundance and $\mathrm{CNO}$ patterns characteristic of dredge-up of CNO cycle products. Ramirez et al. (2000) analyzed 10 stars in the Galactic center and found the mean $[\mathrm{Fe} / \mathrm{H}]$ to be nearly solar, with $[\mathrm{Fe} / \mathrm{H}]=+0.12 \pm 0.22$. Furthermore, the mean $[\mathrm{Fe} / \mathrm{H}]$ of the Galactic center stars was very similar to the mean $[\mathrm{Fe} / \mathrm{H}]$ of $11 \mathrm{cool}$, luminous stars in the solar neighborhood, chosen to have temperatures and luminosities similar to the Galactic center stars. Ramirez et al. (2000) also found that the distribution of $[\mathrm{Fe} / \mathrm{H}]$ in Galactic center stars is 
significantly narrower than the distribution of $[\mathrm{Fe} / \mathrm{H}]$ in the bulge, emphasizing the differences between the stellar populations of the bulge and center.

In this study we analyze the same sample stars as in the Ramirez et al. (2000) study, but with additional sets of high-resolution infrared spectra. All but one target star are members of the Central Cluster and are located at distances of $R_{g}=0.21-2.2 \mathrm{pc}$ (Blum et al. 1996a). In order to sample a different location in the Galactic center, one target (VR 5-7) is a member of the Quintuplet Cluster at $R_{g}=31 \mathrm{pc}$ (Moneti et al. 1994). The goal is to include abundances from $\alpha$-capture elements, in order to begin to probe the abundance distribution patterns in Galactic center stars. The studied elements include carbon, nitrogen, oxygen, and calcium. In order to have a homogeneous and self-consistent analysis of abundance patterns, we also rederived $\mathrm{Fe}$ abundances for the sample stars.

\section{OBSERVATIONS AND DATA REDUCTION}

Observations were made at the $8 \mathrm{~m}$ Gemini South telescope, on Cerro Pachón in northern Chile. Spectra were obtained with the NOAO infrared echelle spectrograph Phoenix (Hinkle et al. 2003) as part of observing programs GS-2002A-DD-1 and GS-2003ADD-2. This spectrograph has a resolution $\lambda / \Delta \lambda \approx R=50,000$ when used, as in these observations, with a slit width of 4 pixels $\left(0.34^{\prime \prime}\right)$. Spectra were obtained on the nights of 2002 May 10 and 11 , June 11 and 13, and 2003 July 29 . Typical image quality estimated on acquisition frames $(1.5$ or $2.3 \mu \mathrm{m})$ was approximately $0.5^{\prime \prime}-1.0^{\prime \prime}$, and some data were taken through thin clouds.

Phoenix spectra were obtained of five Galactic center stars (BSD 72, BSD 114, BSD 124, IRS 11, IRS 19) selected from the sample of Ramirez et al. (2000) and of one solar neighborhood S giant (HD 172804) selected from the sample of Smith \& Lambert $(1985,1986,1990)$. Each star was observed at two grating settings with central wavelengths of $1.5649 \mu \mathrm{m}$ (filter H6420) and $2.3153 \mu \mathrm{m}$ (filter K4308). Stars of spectral types A or B were also observed at an airmass similar to that of each star. The spectra of these hot stars, which are featureless at the wavelengths of interest, were later divided into the spectra of the Galactic center stars to remove telluric absorption features.

Each star was nodded along the slit, and spectra were obtained at two slit positions for background subtraction. In the very crowded stellar field of the Galactic center, the nod spacing was carefully chosen for each star to avoid another star falling into the background position. In several cases, the source was nodded off the slit in order to find a clear sky position. Flat fields were obtained by observing the facility-provided flat-field lamp (GCAL; Phoenix uses a non-standard M3 position in the Gemini instrument support structure).

All spectra were flat-fielded, background subtracted, and corrected for bad pixels. Each Galactic center star spectrum was divided by the appropriate hot star, with the same nod spacing along the slit, to correct for telluric absorption. This also provided some correction for fringing in the spectrograph. Wavelength calibration was done using telluric lines. The two nod positions for each star were reduced separately, rather than co-added, for as long as possible through the analysis as a control on systematic uncertainties such as fringing. No flux calibration was done.

Additional observations of 10 Galactic center stars from the sample of Ramirez et al. (2000) and four solar neighborhood stars from the sample of Smith \& Lambert $(1985,1986,1990)$ were made at the NASA Infrared Telescope Facility $3 \mathrm{~m}$ telescope at the Mauna Kea Observatory. Spectra were obtained with the facility infrared echelle spectrograph, CSHELL, at $R=40,000$

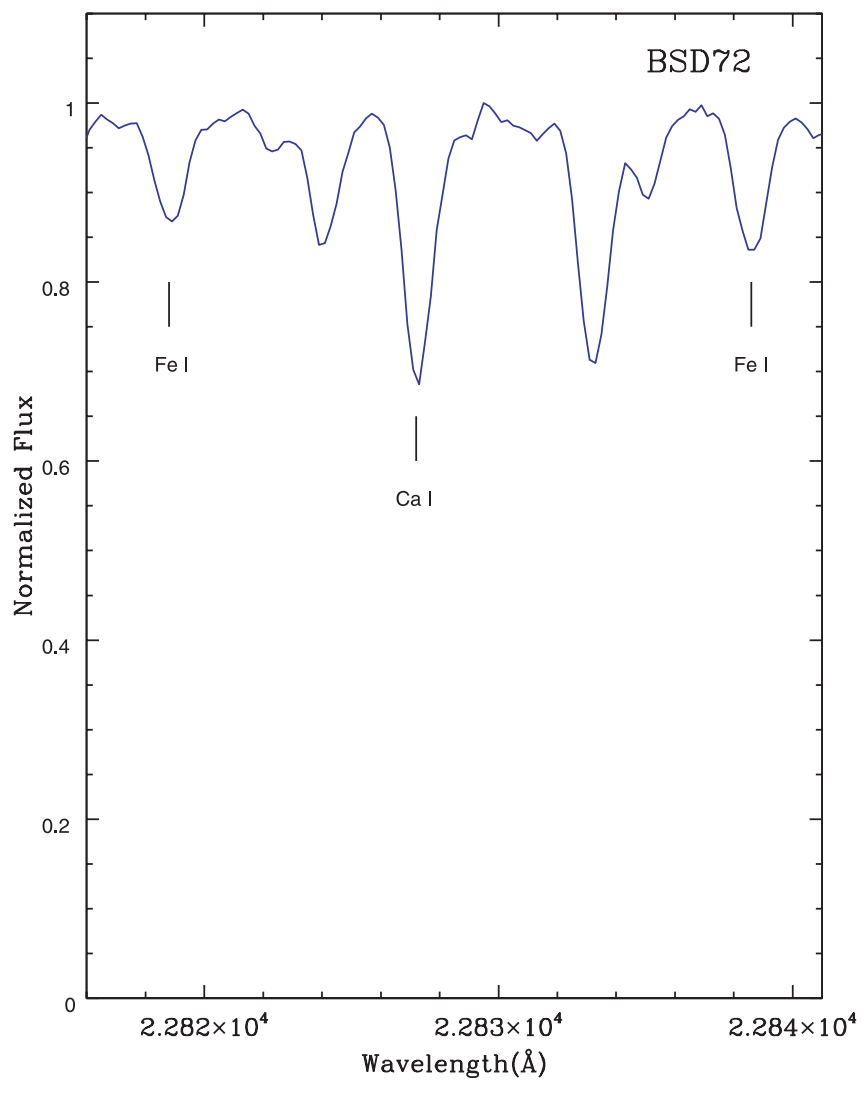

FIG. 1.-Sample spectrum for Galactic center star BSD 72 in the region containing two sample $\mathrm{Fe}_{\mathrm{I}}$ lines (at 22818.8 and $22838.6 \AA$ ) and the Ca I line (at $22827.3 \AA$ ) analyzed in this study.

(Tokunaga et al. 1990). These observations have been previously described by Ramirez et al. (2000); in brief, the observing technique was identical to that described above. Figure 1 shows one sample spectrum obtained with for the Galactic center star BSD 72.

\section{ANALYSIS}

\subsection{Effective Temperatures and Surface Gravities}

Ten Galactic center red stars are analyzed here, with all program stars included in the abundance study by Ramirez et al. (2000). Effective temperatures were derived for these stars by Blum et al. (2003) using spectral indices defined by both $\mathrm{CO}$ and $\mathrm{H}_{2} \mathrm{O}$ absorption. The use of spectral indices is necessitated by the extremely large reddening toward the Galactic center $\left(A_{K} \sim 3\right)$, which renders even infrared colors, such as $J-K$, too uncertain. The absorption indices defined by Blum et al. (2003) are measured relative to spectral regions adjacent to the absorption itself and are thus independent of reddening.

The CO index is defined largely by the $2-0{ }^{12} \mathrm{CO}$ band head at a wavelength of $2.2935 \mu \mathrm{m}$ and is sensitive to both $T_{\mathrm{eff}}$ and luminosity class (i.e., gravity), with the $\mathrm{CO}$ absorption increasing with luminosity for a given temperature. The $\mathrm{H}_{2} \mathrm{O}$ index is composed of a broad absorption feature stretching from the red end of the $H$ band into the blue part of the $K$ band. Blum et al. (2003) measure the $\mathrm{H}_{2} \mathrm{O}$ index from $R=600$ spectra from 1.2 to $2.4 \mu \mathrm{m}$ and define continuum points for their $\mathrm{H}_{2} \mathrm{O}$ index at both the blue end and the red end of the broad $\mathrm{H}_{2} \mathrm{O}$ absorption band to mitigate any sensitivity of the $\mathrm{H}_{2} \mathrm{O}$ index to reddening. The $\mathrm{H}_{2} \mathrm{O}$ absorption in both $\mathrm{M}$ giants and supergiants spans about the same range of values, while Mira variables exhibit 

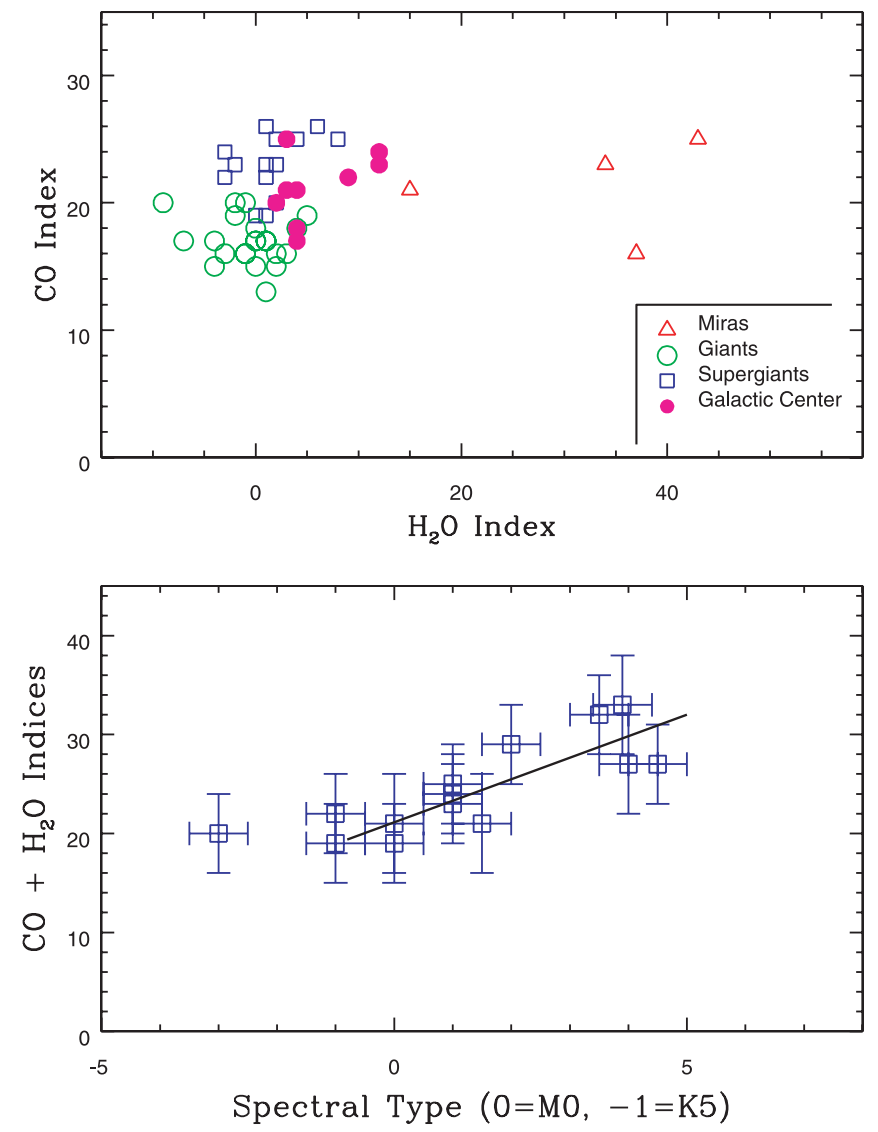

FIG. 2.-Top: $\mathrm{CO}$ and $\mathrm{H}_{2} \mathrm{O}$ absorption indices in various types of cool luminous stars (from Blum et al. 2003). The giants and supergiants segregate quite cleanly in this diagram, with the $\mathrm{CO}$ index increasing with increasing luminosity. The Miras form an extended sequence toward extremely strong $\mathrm{H}_{2} \mathrm{O}$ absorption. The Galactic center stars fall closest to the more luminous giants to supergiants, along a sequence defined by somewhat stronger $\mathrm{H}_{2} \mathrm{O}$ absorption. Bottom: Total $\mathrm{CO}+\mathrm{H}_{2} \mathrm{O}$ absorption versus spectral type for supergiants (from Blum et al. 2003). Between spectral types $\mathrm{M} 0$ to $\mathrm{M} 5$ there is a well-defined increase in $\mathrm{CO}+\mathrm{H}_{2} \mathrm{O}$ toward later spectral types. The straight line is a linear fit to the data from M0 to M5.

considerably stronger absorption by $\mathrm{H}_{2} \mathrm{O} . \mathrm{H}_{2} \mathrm{O}$ is expected to exhibit the opposite change with respect to gravity compared to $\mathrm{CO}$; the feature becomes weaker in stars of the same spectral type but lower gravity (Kleinmann \& Hall 1986). Long-period variables (LPVs, e.g., Mira-type stars) exhibit considerably stronger absorption than normal giants or supergiants owing to the very cool temperatures of LPVs.

A comparison of $\mathrm{CO}$ absorption versus $\mathrm{H}_{2} \mathrm{O}$ absorption is shown in the top panel of Figure 2 for samples of standard giants, supergiants, and Miras (taken from the values in Blum et al. 2003). This type of plot segregates these three stellar groups. Also shown in Figure 2 (top) are the Galactic center stars; these stars largely inhabit the region that both overlaps and is adjacent to the giants and supergiants, with the Galactic center stars tending to have $\mathrm{CO}$ strengths more similar to supergiants but with stronger $\mathrm{H}_{2} \mathrm{O}$. Most of the lower luminosity giants tend to have both lower overall $\mathrm{CO}$ and $\mathrm{H}_{2} \mathrm{O}$ absorption. A simplified view of the Galactic center stars studied here would most closely associate them with luminous giants or supergiants and not much overlap with the Miras. The $\mathrm{CO}$ and $\mathrm{H}_{2} \mathrm{O}$ absorption indices can be used as a way to associate a spectral type with a particular Galactic center star: recall that the extreme and variable reddening into the Galactic center precludes the use of even infrared colors to ascertain reliable effective temperatures.
Blum et al. (2003) relied primarily on a calibration of the $\mathrm{CO}$ index with $T_{\text {eff }}$ defined by $\mathrm{M}$ giants and supergiants, which define two sequences in a $T_{\text {eff }}$-CO plane that are separated by about $300 \mathrm{~K}$ at a given value of $\mathrm{CO}$ absorption (with the supergiants being hotter). In this study, a somewhat different calibration was tried and compared to that from Blum et al. (2003). There is a slight dependence in both the $\mathrm{CO}$ and $\mathrm{H}_{2} \mathrm{O}$ indices with the stellar $\mathrm{C} / \mathrm{O}$ ratios, when the measured $\mathrm{C} / \mathrm{O}$ ratios are taken from Smith \& Lambert (1990). These dependences are caused by the dominant role played by the tightly bound $\mathrm{CO}$ molecule on the partial pressures of not only $\mathrm{CO}$ itself, but also $\mathrm{H}_{2} \mathrm{O}$. The stars analyzed are all of spectral type $\mathrm{M}$, where $\mathrm{C} / \mathrm{O} \leq 1.0$, and for a given $\mathrm{O}$-abundance, the $\mathrm{CO}$ absorption increases with increasing $\mathrm{C} / \mathrm{O}$ ratio. At the same time as the $\mathrm{C} / \mathrm{O}$ increases, the amount of free oxygen decreases, and the subsequent absorption by $\mathrm{H}_{2} \mathrm{O}$ decreases. Thus, there is a slight positive slope of $\mathrm{CO}$ absorption and a slight negative slope of $\mathrm{H}_{2} \mathrm{O}$ as $\mathrm{C} / \mathrm{O}$ increases.

The somewhat modified method for estimating spectral types explored here involves adding the $\mathrm{CO}$ and $\mathrm{H}_{2} \mathrm{O}$ indices as a way of canceling, to some degree, $\mathrm{C} / \mathrm{O}$ trends. The resulting relation between the sum of the $\mathrm{CO}$ and $\mathrm{H}_{2} \mathrm{O}$ indices versus spectral type, for the standard supergiants in Blum et al. (2003), is illustrated in the bottom panel of Figure 2. There is a well-defined relation for spectral types later than $\mathrm{M} 0$ which will include all of the Galactic center stars studied here. A straight line was fit to the absorption index versus spectral type for M0-M5 and this line is also shown in the figure.

The spectral types derived from the $\mathrm{H}_{2} \mathrm{O}+\mathrm{CO}$ indices were then mapped into effective temperatures via a spectral type $-T_{\text {eff }}$ relation as shown in Figure 3. The supergiant points on this plot were taken from the recent analysis of the red supergiant $T_{\text {eff }}$ scale by Levesque et al. (2005). The relation between spectral type and effective temperature is quite well defined. Included in Figure 3 is the relation for M giants of Smith \& Lambert $(1985,1986,1990)$ coupled to the accurate spectral types from Yamashita (1971). These relations have involved no color information but rely mainly on the TiO bands in the visible and seem to present very similar relations for both giants and supergiants. The two sequences appear to begin to separate at spectral types later than about M4, but almost no supergiants are found later than about M5 anyway. The resulting relation for the supergiants was used to estimate the spectral types of the Galactic center stars, based on their measured $\mathrm{CO}$ and $\mathrm{H}_{2} \mathrm{O}$ indices; this relation was selected based on the luminosities of the Galactic center stars, as discussed above.

The final $T_{\text {eff }}$ adopted was an average of that derived here and the temperature from Blum et al. (2003). The comparison between the two estimates is good, with the mean and standard deviation being $\Delta$ (this study - Blum et al. $)=+72 \pm 138 \mathrm{~K}$. The scatter of $\sim \pm 140 \mathrm{~K}$ found between the two $T_{\text {eff }}$ scales can be compared to the expected inherent uncertainties in the $T_{\text {eff }}$ scale adopted here based on errors in the fundamental quantities. The error in the scale of $\mathrm{H}_{2} \mathrm{O}+\mathrm{CO}$ is about \pm 5 , which translates to an uncertainty in spectral type of \pm 1.5 subtypes (Fig. 2). This uncertainty in spectral type leads to an expected error of $\sim \pm 150 \mathrm{~K}$ from the relation plotted in Figure 3. The effective temperatures adopted for the studied stars are presented in Table 1 (Table 2 refers to the comparison disk stars described below).

Surface gravities (noted as $\log g$ ) for the Galactic center stars are also listed in Table 1 . These values were derived by combining the effective temperatures with the absolute bolometric magnitudes taken from Blum et al. (2003). With the stars plotted in a $M_{\text {bol }}$ versus $T_{\text {eff }}$ diagram, stellar evolutionary model tracks are added and each program stellar mass is estimated (Table 1, col. [7]). 


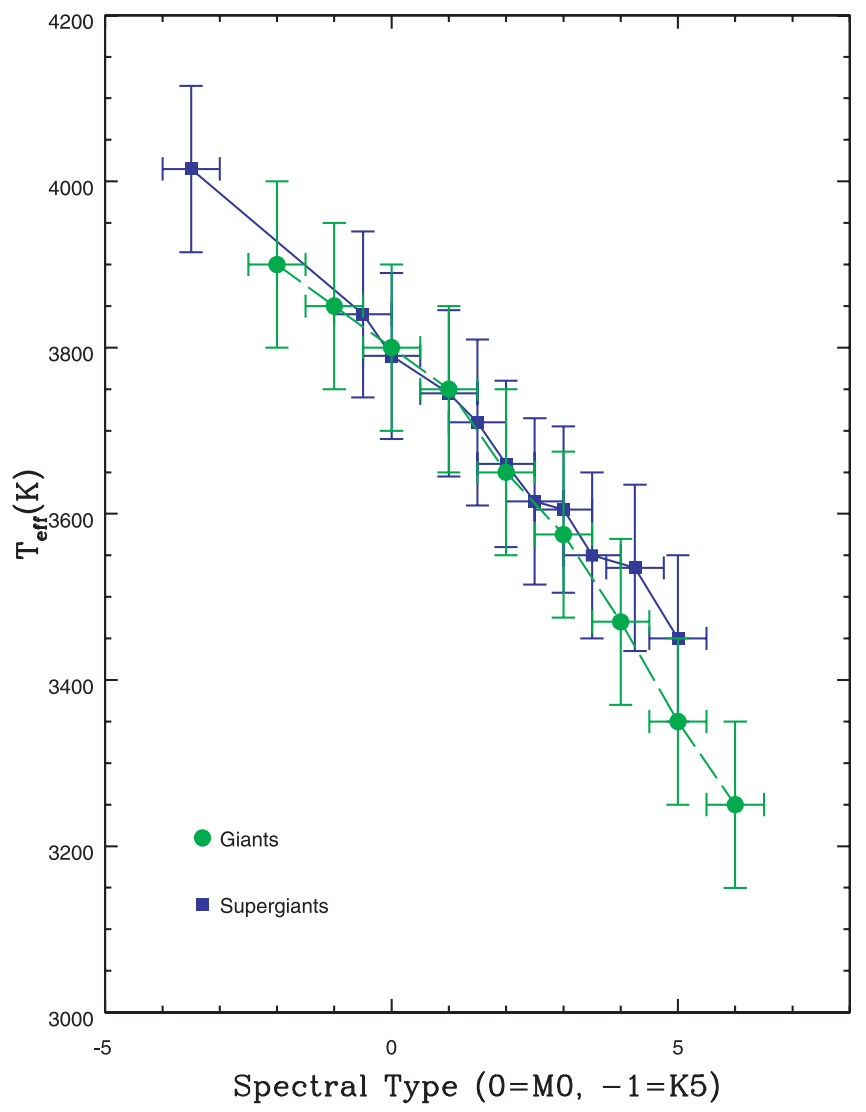

FIG. 3.-Effective temperatures as a function of spectral type for both giants and supergiants. The supergiant scale is taken from Levesque et al. (2005), while the giant scale is defined by effective temperatures from the studies of Smith \& Lambert $(1985,1986,1990)$ coupled to the spectral types from Yamashita (1971). There is a tight relation between $T_{\text {eff }}$ and spectral type, with the giant and supergiant scales not so different when defined in terms of spectral type. The error bars illustrate uncertainties of $\pm 100 \mathrm{~K}$ in $T_{\text {eff }}$ and \pm 0.5 in spectral type.

The stellar model tracks are taken from the model grids from Salasnich et al. (2000) ${ }^{2}$ and slightly "supersolar" metallicities are used $(Z=0.019$ or $[m / H]=+0.1)$, which correspond fairly closely to the final metallicities derived for the Galactic center stars. The $M_{\mathrm{bol}}-T_{\text {eff }}$ diagram is illustrated in Figure 4 . The Galactic

\footnotetext{
2 These can be found online at http://pleiadi.pd.astro.it.
}

center stars are shown as the circles. Representative stellar model evolutionary tracks are also shown with the model mass labeled for each track.

As a test of the analysis techniques used for the Galactic center stars, four bright "local" disk M giants and one S giant were analyzed in the same way, using the same wavelength regions. The five stars were HR 6146, HR 6702, HR 7442, HR 8062, and HD 172804. Their spectral types from Yamashita (1971) were used with the spectral type- $T_{\text {eff }}$ relation (Fig. 3 ) to determine their effective temperatures. All five of these stars have measured Hipparcos parallaxes, which were used to determine distances and absolute $V$ and $K$ magnitudes. Given their $V-K$ colors, the $K$-band bolometric corrections from Bessel et al. (1998) were applied to determine $M_{\text {bol }}$ for each M giant or S star. In this way, surface gravities were derived in the same way as for the Galactic center stars in an $M_{\text {bol }}-T_{\text {eff }}$ diagram and the estimated masses from the Padua evolutionary tracks; these stars are represented as squares in Figure 4. The derived stellar parameters for the bright disk $M$ giants are listed in Table 2.

The "nearby" giants cluster around $M_{\text {bol }} \sim-4.1$ to -4.7 , and this roughly overlaps the lower luminosity members of the Galactic center sample. The effective temperatures also overlap between the two samples. Thus, no large systematic differences are expected in the derived abundances from the two samples. The bright $\mathrm{M}$ giants constitute a valuable control sample in interpreting the abundance patterns in the Galactic center stars.

Yusef-Zadeh \& Morris (1991) and Serabyn et al. (1991) discovered that the outer envelope of IRS 7 is ionized and that it has a tail of ionized gas. This has been interpreted as due to the interaction of the winds from hot stars in IRS 16 and the mass-loss wind from IRS 7 (Serabyn et al. 1991; Yusef-Zadeh \& Melia 1992). There is no evidence from our high-resolution infrared spectra that the presence of a tail of ionized gas affects in any measurable way the formation of the photospheric spectra in IRS 7 (see also Carr et al. 2000). Other wind-wind interactions have been discovered in the Central Cluster, appearing as bow shocks around suspected hot stars in the mid-infrared (Tanner et al. 2005), but none of the other stars in our Galactic center sample are among these sources. The photospheric absorption lines observed at high spectral resolution from $\mathrm{CO}, \mathrm{OH}, \mathrm{CN}, \mathrm{Fe} \mathrm{I}$, and $\mathrm{Ca}$ I, for the stars in our Galactic center sample, look normal when compared to the Galactic disk control sample. In addition, all of our Galactic center sample stars have $H$ - and $K$-band spectra at $R=600-1200$ (Blum et al. 1996b, 2003), and some have $K$-band spectra at $R=2500$ (Sellgren et al. 1987). The only

TABLE 1

Galactic Center Stars and Stellar Parameters

\begin{tabular}{|c|c|c|c|c|c|c|c|}
\hline $\begin{array}{c}\text { Star } \\
\text { (1) }\end{array}$ & $\begin{array}{l}T_{\text {eff }} \\
(\mathrm{K}) \\
(2)\end{array}$ & $\begin{array}{c}\log g \\
\left(\mathrm{~cm} \mathrm{~s}^{-2}\right) \\
(3)\end{array}$ & $\begin{array}{c}\xi \\
\left(\mathrm{km} \mathrm{s}^{-1}\right) \\
(4)\end{array}$ & $\begin{array}{c}\zeta \\
\left(\mathrm{km} \mathrm{s}^{-1}\right) \\
(5)\end{array}$ & $\begin{array}{c}M_{\text {bol }} \\
\text { (6) }\end{array}$ & $\begin{array}{c}\text { Mass } \\
\left(M_{\odot}\right) \\
(7)\end{array}$ & $\begin{array}{c}R_{g}^{\mathrm{a}} \\
(\mathrm{pc}) \\
(8)\end{array}$ \\
\hline BSD $72 \ldots \ldots \ldots \ldots$ & 3880 & 0.8 & 2.1 & $10-12$ & -4.54 & 5 & 1.6 \\
\hline 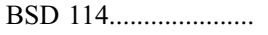 & 3150 & -0.3 & 2.6 & $12-16$ & -5.27 & 2 & 2.1 \\
\hline BSD 124 ........................ & 3735 & 0.4 & 2.5 & $12-14$ & -5.50 & 6.5 & 1.9 \\
\hline BSD 129 & 3800 & 0.5 & 2.5 & $9-14$ & -5.33 & 6.5 & 2.2 \\
\hline BSD $140 \ldots \ldots \ldots \ldots \ldots$ & 3200 & 0.0 & 3.1 & $14-16$ & -4.62 & 2 & 2.0 \\
\hline IRS $7 \ldots \ldots \ldots$ & 3650 & -0.5 & 3.2 & 25 & -9.00 & 22 & 0.21 \\
\hline IRS $11 \ldots \ldots \ldots \ldots$ & 3625 & 0.3 & 2.3 & $11-15$ & -5.04 & 4.5 & 0.62 \\
\hline IRS $19 \ldots \ldots \ldots \ldots \ldots$ & 3850 & 0.1 & 3.0 & $13-15$ & -7.28 & 15 & 1.0 \\
\hline IRS $22 \ldots \ldots \ldots \ldots \ldots$ & 3750 & 0.2 & 2.3 & $11-16$ & -6.49 & 10 & 1.0 \\
\hline VR $5-7 \ldots \ldots \ldots \ldots \ldots$ & 3600 & -0.15 & 2.6 & $14-16$ & -7.62 & 14 & 31.0 \\
\hline
\end{tabular}

${ }^{\text {a }}$ Projected galactocentric distance. 
TABLE 2

Disk Stars and Stellar Parameters

\begin{tabular}{|c|c|c|c|c|c|c|}
\hline $\begin{array}{c}\text { Star } \\
\text { (1) }\end{array}$ & $\begin{array}{l}T_{\text {eff }} \\
(\mathrm{K}) \\
(2)\end{array}$ & $\begin{array}{c}\log g \\
\left(\mathrm{~cm} \mathrm{~s}^{-2}\right) \\
(3)\end{array}$ & $\begin{array}{c}\xi \\
\left(\mathrm{km} \mathrm{s}^{-1}\right) \\
(4)\end{array}$ & $\begin{array}{c}\zeta \\
\left(\mathrm{km} \mathrm{s}^{-1}\right) \\
(5)\end{array}$ & $\begin{array}{c}M_{\text {bol }} \\
\text { (6) }\end{array}$ & $\begin{array}{c}\text { Mass } \\
\left(M_{\odot}\right) \\
(7)\end{array}$ \\
\hline 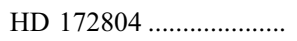 & 3400 & 0.3 & 2.9 & 10 & -4.7 & 2.2 \\
\hline 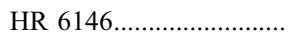 & 3250 & 0.14 & 2.1 & $10-12$ & -4.1 & 1.5 \\
\hline 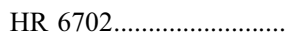 & 3300 & 0.14 & 2.1 & $9-11$ & -4.1 & 2 \\
\hline HR $7442 \ldots \ldots \ldots \ldots \ldots \ldots$ & 3450 & 0.09 & 2.2 & $8-12$ & -4.4 & 2.5 \\
\hline HR $8062 \ldots \ldots \ldots \ldots \ldots$ & 3450 & 0.31 & 2.3 & $8-12$ & -4.3 & 2 \\
\hline
\end{tabular}

differences from normal $\mathrm{M}$ supergiant/asymptotic giant branch (AGB) star spectra that have been noted can be attributed to abundance patterns that we infer later from our high-resolution spectroscopy.

\subsection{Microturbulent Velocities}

The microturbulent velocity parameter $(\xi)$ for the program stars was obtained using a two-step process. First, the measured equivalent widths of the $\mathrm{Fe}$ I lines were used to derive an initial estimate of the microturbulence from the requirement that the $\mathrm{Fe}$ I equivalent widths be independent of the derived $\mathrm{Fe}$ abundances. This method would be sufficient if the lines were unblended. The Galactic center stars under analysis, however, have fairly crowded spectra, and one must check whether contributions from $\mathrm{CN}$ lines and other blending lines influence the $\mathrm{Fe}$ I equivalent widths. As a second step, therefore, the line list in $\S 3.3 .2$

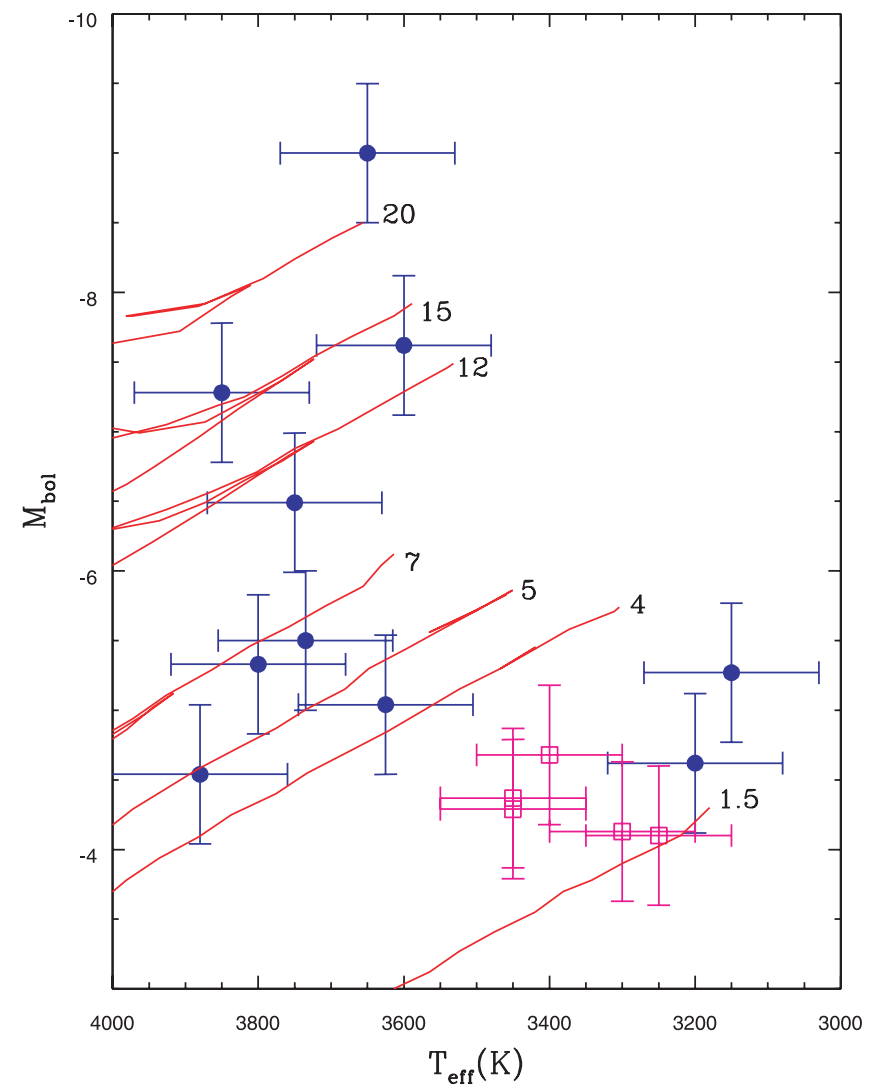

FIG. 4.-Absolute bolometric magnitudes and effective temperatures for the Galactic center stars (blue circles) and the brighter disk "standard" M stars (magenta squares). Model stellar evolutionary tracks of representative masses are plotted as the solid red curves, with the model stellar mass indicated by each track. The stellar model tracks are from Salasnich et al. (2000). was used to compute a grid of synthetic spectra for a range of microturbulent velocities bracketing the initial microturbulence value defined in the first step. The observed and synthetic spectra were compared to find the final microturbulence which best fit each star. The values presented in Tables 1 and 2 are the results from such a fine-tuning of $\xi$.

A direct comparison of our microturbulent velocities with the values derived by Ramirez et al. (2000) shows good agreement (this study; Ramirez et al. 2000): $\Delta \xi=-0.0 \pm 0.3 \mathrm{~km} \mathrm{~s}^{-1}$. In addition, Carr et al. (2000) obtained $\xi=3.0 \mathrm{~km} \mathrm{~s}^{-1}$ for IRS 7, which is within the errors of our adopted value $\left(3.2 \pm 0.3 \mathrm{~km} \mathrm{~s}^{-1}\right)$ for this star. In Figure 5 the microturbulent velocities and surface gravities are compared with results from several different studies in

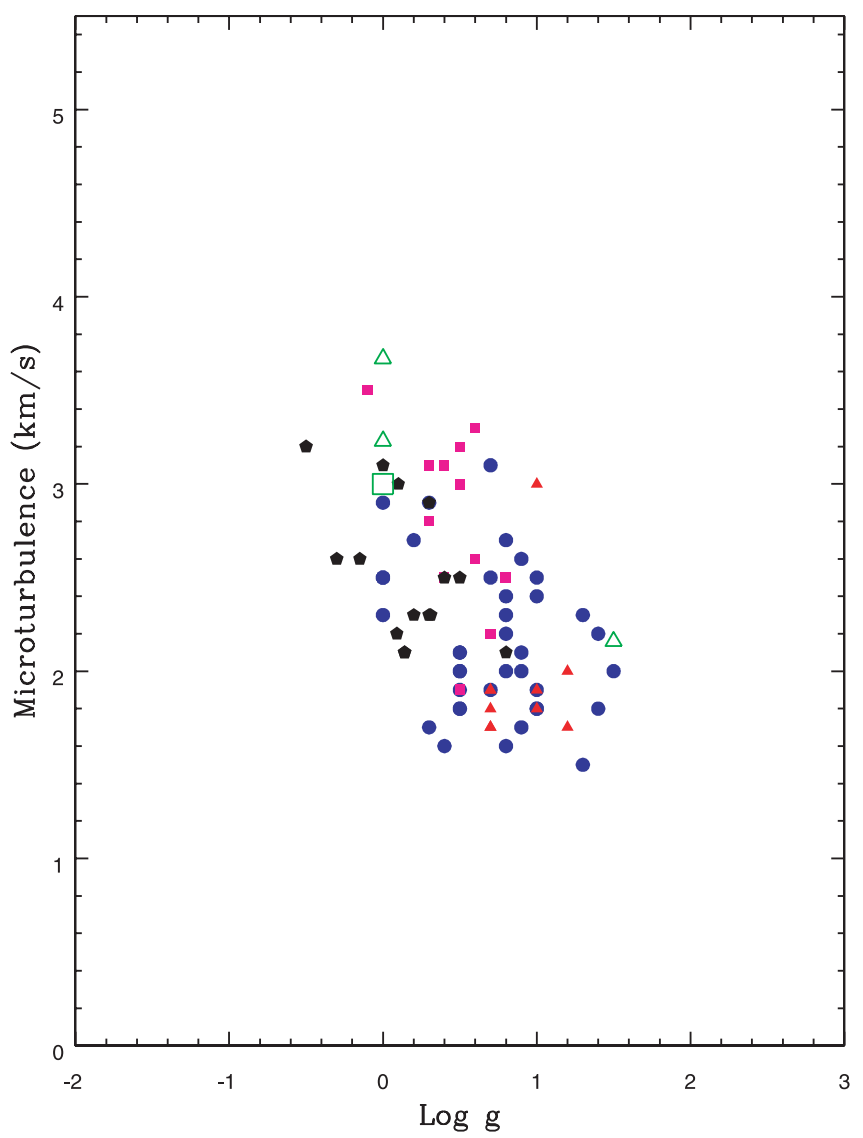

FIG. 5.-Microturbulent velocities and surface gravities for a number of spectroscopic analyses of red giants and supergiants. The results from this study for both Galactic center and disk stars are represented by black pentagons. The other studies include Smith \& Lambert (1986, 1990; filled blue circles), Carr et al. (2000; open green triangles), Smith et al. (2000; filled magenta squares), Smith et al. (2002; filled red triangles), and Lambert et al. (1984; open green square; $\alpha$ Ori). 
the literature. Although there is significant scatter of the points in the figure, the general trend can be described as a rough correlation between the microturbulence parameter and the surface gravity of the star (as discussed in, e.g., Ramirez et al. 2000). In particular, we note that the microturbulent velocities in this study (Fig. 5, black pentagons) generally fall within the range of values defined for those stars with similar surface gravities; and the well-studied supergiant $\alpha$ Ori (Fig. 5, open green square) falls within the scatter of points defined by the Galactic center stars which have supergiant-like luminosities.

\subsection{Abundances}

The abundance analysis in this study was carried out via comparisons of synthetic spectra with the observed ones. The model atmospheres adopted for the studied late-type giants and supergiants are from the grid of spherical models by Plez (1992) and Plez et al. (1992). The program MOOG (Sneden 1973) was used in order to compute LTE synthetic spectra given the required input model atmosphere defined by the parameters of effective temperature $\left(T_{\text {eff }}\right)$, surface gravity $(\log g)$, and microturbulent velocity $(\xi)$. The synthetic spectra were convolved with both a Gaussian function, representing the instrumental profile, as well as a macroturbulent velocity function (Gray 1992) to properly match the observed profiles of the spectral lines via minimizing the residuals between the observed and synthetic profiles. The macroturbulent velocities adopted in the calculations are presented in column (5) of Tables 1 and 2.

\subsubsection{Carbon, Nitrogen, and Oxygen}

The $\mathrm{C}, \mathrm{N}$, and $\mathrm{O}$ abundances in this study were derived from observations of $\mathrm{OH}$ and $\mathrm{CN}$ molecular lines present in the $H$-band region, as well as $\mathrm{CO}$ lines present in the $K$-band region of the Phoenix spectra obtained for three Galactic center stars: BSD 72, BSD 124, and IRS 19. In order to obtain a consistent solution for the molecular equilibrium involving $\mathrm{CN}, \mathrm{CO}$, and $\mathrm{OH}$ molecules, we first synthesized the $\mathrm{OH}$ molecular lines in the $H$-band region. The $\mathrm{OH}$ lines analyzed here are listed in Table 3 and, except for the $\mathrm{OH}$ transition at $15627 \AA$, these were also analyzed in a recent study of $\mathrm{K}$ and $\mathrm{M}$ giants of the Galactic bulge (Cunha \& Smith 2006). These $\mathrm{OH}$ lines analyzed in bulge red giants gave generally consistent oxygen abundances when compared to the results from the optical study by Fulbright et al. (2006), which analyzed the forbidden neutral oxygen ([O I ] ) in the same bulge stars. In the more luminous Galactic center stars studied here, however, the stronger $\mathrm{OH}$ lines become, in some cases, extremely strong and therefore very sensitive to the microturbulence parameter. Such dependence on microturbulence renders these lines too uncertain to be useful as abundance indicators. The weaker $\mathrm{OH}$ line at $15627 \AA$, however, could be measured in all Galactic center stars and served as the sole abundance indicator in IRS 19 (which is the most luminous Galactic center star of the trio) and BSD 114 (only observed at one $H$-band setting).

A simultaneous solution for $\mathrm{CO}$ was obtained, at the same time as $\mathrm{OH}$ was analyzed, from computations of synthetic spectra of the first overtone vibration-rotation molecular lines of $\mathrm{CO}$ around $23100 \AA$. The CO lines used are listed in Table 3. The $\log g f$ values and excitation potentials for the transitions were obtained from Goorvitch (1994). We note, again, that because the Galactic center stars are more luminous when compared to the bulge giants, the stronger CO lines in the spectra are so strong that they are extremely sensitive to the microturbulence parameter. The application of the simple requirement of searching for an agreement between the abundances of strong and weak $\mathrm{CO}$ lines in the spectra would demand a higher microturbulent velocity, around
TABLE 3

Spectral Lines (Analyzed via Spectrum Synthesis)

\begin{tabular}{|c|c|c|}
\hline $\begin{array}{c}\lambda \\
(\AA)\end{array}$ & $\begin{array}{c}\chi \\
(\mathrm{eV})\end{array}$ & $\log g f$ \\
\hline \multicolumn{3}{|c|}{ Fe I } \\
\hline $22381.27 \ldots \ldots \ldots \ldots \ldots \ldots \ldots \ldots$ & 5.844 & -1.511 \\
\hline $22386.90 \ldots \ldots \ldots \ldots$ & 5.033 & -0.224 \\
\hline $22391.22 \ldots \ldots \ldots$ & 5.320 & -1.625 \\
\hline $22398.98 \ldots \ldots \ldots$ & 5.099 & -1.134 \\
\hline 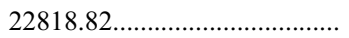 & 5.792 & -0.992 \\
\hline 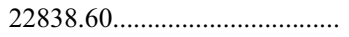 & 5.099 & -1.222 \\
\hline \multicolumn{3}{|c|}{$\mathrm{Ca} \mathrm{I}$} \\
\hline 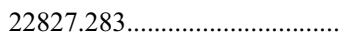 & 4.624 & -0.246 \\
\hline \multicolumn{3}{|c|}{${ }^{12} \mathrm{C}^{14} \mathrm{~N}$} \\
\hline $15530.987 \ldots \ldots$ & 0.89 & -1.519 \\
\hline $15544.501 \ldots \ldots$ & 1.15 & -1.146 \\
\hline 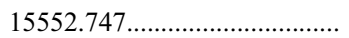 & 0.90 & -1.680 \\
\hline 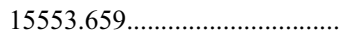 & 1.08 & -1.285 \\
\hline 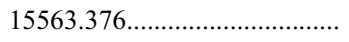 & 1.15 & -1.141 \\
\hline \multicolumn{3}{|c|}{${ }^{16} \mathrm{OH}$} \\
\hline $15535.489 \ldots \ldots$ & 0.51 & -5.233 \\
\hline $15560.271 \ldots \ldots$ & 0.30 & -5.307 \\
\hline $15568.807 \ldots \ldots$. & 0.30 & -5.270 \\
\hline $15572.111 \ldots \ldots$. & 0.30 & -5.183 \\
\hline $15627.290 \ldots \ldots \ldots \ldots \ldots \ldots \ldots$ & 0.89 & -5.435 \\
\hline 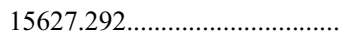 & 0.89 & -5.435 \\
\hline 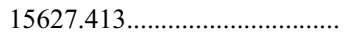 & 0.54 & -5.198 \\
\hline \multicolumn{3}{|c|}{${ }^{12} \mathrm{C}^{16} \mathrm{O}$} \\
\hline $23108.742 \ldots \ldots$ & 1.51 & -4.907 \\
\hline 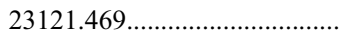 & 1.55 & -4.900 \\
\hline 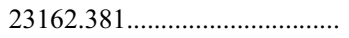 & 1.66 & -4.879 \\
\hline 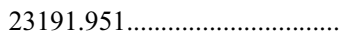 & 1.74 & -4.857 \\
\hline
\end{tabular}

$3-4.5 \mathrm{~km} \mathrm{~s}^{-1}$, and in some cases even higher. The formation of such strong CO lines was investigated, and it was found that the cores of these lines form extremely high (i.e., at low values of optical depth) in the stellar atmospheres of these cool and luminous stars. Such lines have large abundance uncertainties attached to them due to sensitivity to structure in the upper layers of the model atmosphere, for instance, the choice of the optical-depth boundary of the model atmosphere. In this study the strong $\mathrm{CO}$ lines were rejected from the analysis, and we relied on the weaker CO lines (from Table 3) as abundance indicators.

Finally, the weaker $\mathrm{CN}$ lines in the $H$-band spectral region around $15540 \AA$ (Table 3) were synthesized in order to derive nitrogen abundances for the Galactic center stars. This is the same spectral region (covered by some of the Phoenix spectra) that was analyzed in the bulge study by Cunha \& Smith (2006); that paper is referred to for details on the construction of the $\mathrm{CN}$ line list. The $\mathrm{CN}$ lines are considerably weaker and somewhat more uncertain to measure in the cases of spectra with lower signal-to-noise ratios $(\mathrm{S} / \mathrm{N})$. They are not affected, however, by the same problems as were found for the stronger $\mathrm{OH}$ and CO lines.

\subsubsection{Calcium and Iron}

Elemental abundances were derived from atomic lines of $\mathrm{Ca}$ I and $\mathrm{Fe}_{\mathrm{I}}$ in addition to CNO. One suitable line of Ca I (at $22827 \AA$ ) 
TABLE 4

Abundances

\begin{tabular}{|c|c|c|c|c|c|}
\hline Star & $A\left({ }^{12} \mathrm{C}\right)$ & $A\left({ }^{14} \mathrm{~N}\right)$ & $A\left({ }^{16} \mathrm{O}\right)$ & $A(\mathrm{Fe})$ & $A(\mathrm{Ca})$ \\
\hline BSD $72 \ldots \ldots \ldots \ldots$ & 8.44 & 8.20 & 8.80 & 7.51 & 6.66 \\
\hline BSD $114 \ldots \ldots \ldots \ldots$ & $\ldots$ & $\ldots$ & 8.95 & 7.57 & 6.60 \\
\hline BSD $124 \ldots \ldots \ldots \ldots$ & 8.45 & 8.48 & 9.30 & 7.61 & 7.00 \\
\hline BSD $129 \ldots \ldots \ldots \ldots$ & $\ldots$ & $\ldots$ & $\ldots$ & 7.67 & 6.76 \\
\hline BSD $140 \ldots \ldots \ldots \ldots$ & $\ldots$ & $\ldots$ & $\ldots$ & 7.52 & 6.50 \\
\hline IRS $7 \ldots \ldots \ldots \ldots \ldots$ & $\ldots$ & $\ldots$ & $\ldots$ & 7.66 & 6.86 \\
\hline IRS $11 \ldots \ldots \ldots \ldots \ldots$ & 8.40 & $\ldots$ & 9.00 & 7.53 & 6.60 \\
\hline IRS $19 \ldots \ldots \ldots \ldots$ & 8.54 & 8.33 & 9.15 & 7.63 & 6.66 \\
\hline IRS $22 \ldots \ldots \ldots \ldots . . . . . . .$. & $\ldots$ & $\ldots$ & $\ldots$ & 7.57 & 6.93 \\
\hline VR $5-7 \ldots \ldots \ldots \ldots$ & $\ldots$ & $\ldots$ & $\ldots$ & 7.60 & 6.51 \\
\hline HD $172804 \ldots \ldots \ldots$. & 8.50 & $\ldots$ & 8.55 & $\ldots$ & $\ldots$ \\
\hline HR $6146 \ldots \ldots \ldots \ldots . . . . .$. & $\ldots$ & $\ldots$ & $\ldots$ & 7.47 & 6.20 \\
\hline HR $6702 \ldots \ldots \ldots \ldots . . . .$. & $\ldots$ & $\ldots$ & $\ldots$ & 7.42 & 6.18 \\
\hline HR $7442 \ldots \ldots \ldots \ldots . . . .$. & $\ldots$ & $\ldots$ & $\ldots$ & 7.22 & 6.08 \\
\hline HR $8062 \ldots \ldots \ldots \ldots . . . . .$. & $\ldots$ & $\ldots$ & $\ldots$ & 7.31 & 6.31 \\
\hline
\end{tabular}

Note.-Abundance is defined as $A(\mathrm{X})=\log [n(\mathrm{X}) / n(\mathrm{H})]+12$.

and several $\mathrm{Fe}$ I lines were used as abundance indicators, all in the $K$-band spectra of the program stars. We also investigated the possibility of analyzing the Ti I transition at $15543 \AA$ (which was previously studied in Cunha \& Smith 2006), but this neutral titanium line was extremely strong in all the observed spectra of the Galactic center stars and therefore not useful in an abundance analysis.

The sample of Fe I lines for which synthetic spectra were computed in this study is assembled in Table 3; this is the same sample as analyzed previously by Ramirez et al. (2000). The line list for the computation of synthetic spectra was the same as in Ramirez et al. (2000), with the only difference being that we derived new solar $g f$-values for all $\mathrm{Fe}$ I and $\mathrm{Ca}$ I lines. The solar abundances adopted in the calculations were $A(\mathrm{Fe})_{\odot}=7.45$ and $A(\mathrm{Ca})_{\odot}=$ 6.30 (Asplund et al. 2005). The derived solar $g f$-values (Table 3 ) were then checked via an analysis of the $\mathrm{K}$ giant Arcturus, yielding results consistent with published abundances for this star (e.g., Smith et al. 2000). Use of the $22827 \AA \mathrm{Ca}$ I line with the solar $g f$-value yielded an abundance of $A(\mathrm{Ca})=5.86$ for $\alpha$ Boo, compared to the average of $5.75 \pm 0.15$ from Smith et al. (2000), who used a set of optical $\mathrm{Ca}$ I lines with laboratory $g f$-values.

\subsection{Abundance Uncertainties}

Table 4 presents the abundance results for the studied elements $\mathrm{C}, \mathrm{N}, \mathrm{O}, \mathrm{Ca}$, and $\mathrm{Fe}$, while in Table 5 are summarized the corresponding uncertainties in the obtained abundances. The abundance uncertainties were calculated by modifying the stellar parameters of the model atmospheres by amounts corresponding to what are expected to be maximum uncertainties in the effective temperatures $\left(\delta T_{\text {eff }}= \pm 200 \mathrm{~K}\right)$, surface gravities $(\delta \log g=$ $\pm 0.3 \mathrm{dex})$, and microturbulent velocities $\left(\delta \xi= \pm 0.3 \mathrm{~km} \mathrm{~s}^{-1}\right)$. Each one of these parameters was varied independently, adopting as a starting model atmosphere one with $T_{\text {eff }}=3600 \mathrm{~K}$, $\log g=0.5$, and microturbulence $(\xi)=2.2 \mathrm{~km} \mathrm{~s}^{-1}$. The total uncertainty for each element (presented in Table 5, col. [5]) is the sum in quadrature of the individual errors. The sensitivities are different for the different lines, resulting from differing typical line strengths, different ionization or dissociation energies, and different excitation energies. The combination of all errors in Table 5 gives maximum uncertainties of about 0.15 dex for calcium and iron, around $0.2-0.25$ dex for carbon and nitrogen, and around 0.3 dex for oxygen.

In addition to the sources of uncertainties discussed above, it is important to note that non-LTE effects in the Galactic center stars have not been evaluated, and therefore, the abundances derived here may suffer from systematic errors due to non-LTE effects. For the molecules studied here, especially for the $\mathrm{CO}$ and $\mathrm{OH}$ vibration-rotation transitions, the non-LTE departures are expected to be small due to a combination of small transition probabilities (i.e., longer lifetimes), which allow for thermalizing collisions, and the dilute UV radiation fields in cool supergiants. In the case of the atomic lines from $\mathrm{Fe}$ I and $\mathrm{Ca}$ I, their excitation potentials are high enough and the depth of formation is deep enough such that non-LTE effects should not be large.

There is evidence in the abundance results suggesting that non-LTE effects are probably not large. The four disk $M$ giants analyzed for $\mathrm{Fe}$ and $\mathrm{Ca}$ yield values of $[\mathrm{Ca} / \mathrm{Fe}]$ and $[\mathrm{Fe} / \mathrm{H}]$ that fall closely along the disk relation derived from other types of stars (see the discussion in $\S 4.3$ and Fig. 7); this would not be expected if there were measurable effects from non-LTE. In the case of oxygen, the disk S star HD 172804 was observed in the $\mathrm{OH}$ lines and an oxygen abundance of $A\left({ }^{16} \mathrm{O}\right)=8.55$ was derived, in good agreement with its previously published value of 8.50 (Smith \& Lambert 1990). Finally, both oxygen and calcium are produced mostly in supernovae Type II (SNe II), and the abundance ratio of $\mathrm{Ca} / \mathrm{O}$ will be similar for $\mathrm{SNe}$ II with similar metallicity. Since Ramirez et al. (2000) find that the Galactic center iron abundances are close to solar, the values of $\mathrm{Ca} / \mathrm{O}$ in near-solar-metallicity stars might be expected to be mirrored in the Galactic center stars. Indeed, this turns out to be the case; the average value of $[\mathrm{Ca} / \mathrm{O}]$ in the five Galactic center stars for which both calcium and oxygen were measured is $[\mathrm{Ca} / \mathrm{O}]=$ $+0.06 \pm 0.13$. This can be compared to the recent results from Reddy et al. (2003) for 56 near-solar-metallicity F and G dwarfs of $[\mathrm{Ca} / \mathrm{O}]=-0.03 \pm 0.11$. Such close correspondence in very different types of stars is not a coincidence and demonstrates that the abundances derived here for Galactic center stars are not subject to large non-LTE effects (or other unspecified physical processes).

TABLE 5

Abundance UnCertainties

\begin{tabular}{|c|c|c|c|c|}
\hline $\begin{array}{l}\text { Element } \\
\text { (1) }\end{array}$ & $\delta T=\underset{(2)}{+200 \mathrm{~K}}$ & $\delta \log g=+0.3 \mathrm{dex}$ & $\delta \xi=+0.3 \mathrm{~km} \mathrm{~s}^{-1}$ & $\begin{array}{l}\Delta^{\mathrm{a}} \\
(5)\end{array}$ \\
\hline$\Delta^{12} \mathrm{C} \ldots \ldots \ldots \ldots$ & +0.02 & +0.14 & -0.12 & \pm 0.22 \\
\hline$\Delta^{14} \mathrm{~N}$ & +0.20 & -0.11 & -0.05 & \pm 0.25 \\
\hline$\Delta^{16} \mathrm{O}$ & +0.30 & -0.05 & -0.12 & \pm 0.33 \\
\hline$\Delta \mathrm{Ca} \ldots \ldots \ldots \ldots \ldots \ldots$ & +0.10 & +0.02 & -0.11 & \pm 0.15 \\
\hline$\Delta \mathrm{Fe}$ & -0.04 & +0.07 & -0.11 & \pm 0.15 \\
\hline
\end{tabular}

${ }^{\text {a }}$ Total uncertainty is defined as $\left[(\delta T)^{2}+(\delta \log g)^{2}+(\delta \xi)^{2}\right]^{1 / 2}$. 


\section{DISCUSSION}

With the assembled set of elemental abundances from $\mathrm{Fe}$ and $\mathrm{Ca}$ in all program stars, along with the ${ }^{12} \mathrm{C},{ }^{14} \mathrm{~N}$, and ${ }^{16} \mathrm{O}$ abundances in a subset of stars, the abundance distributions can begin to provide initial insights into both the nature of the evolved stars themselves (internal stellar evolution) and the type of chemical evolution that has taken place in the inner central $60 \mathrm{pc}$ of our Galaxy. This discussion focuses first on the $\mathrm{Fe}$ abundances, followed by $\mathrm{C}, \mathrm{N}$, and $\mathrm{O}$ taken together, and then both $\mathrm{O}$ and $\mathrm{Ca}$ as typifying the $\alpha$-elements. With an initial picture of the nature of the chemical evolution in the Galactic center in-hand, the abundance distributions are examined in light of Galactic disk abundance gradients as extrapolated into the center.

\subsection{The Iron Abundance Distribution in the Galactic Center}

Iron is produced in both $\mathrm{SNe}$ Ia and SNe II and historically has been associated with the overall metallicity of a stellar population. Its abundance is also well-defined here by six lines, so this discussion begins with Fe. The Fe results obtained in this study are fully consistent with those derived by Ramirez et al. (2000) and Carr et al. (2000). The average Fe abundance obtained for the sample of 10 stars is slightly higher (by $\sim 0.10-0.15$ dex) than the solar abundance of $A(\mathrm{Fe})=7.45$ recommended by Asplund et al. (2005). The mean value is $A(\mathrm{Fe})=7.59 \pm 0.06$, where the standard deviation represents the scatter among the line-by-line values and probably underestimates the true uncertainties in the derived Fe abundances (the abundance uncertainty estimated for iron is in Table 5 and is roughly $0.15 \mathrm{dex}$ ). This value is quite a bit larger than the actual scatter in the individual abundances, showing that systematic errors dominate the error budget.

Although a relatively small number of stars are analyzed in this study, we note the small abundance range found for iron [from 7.51 to 7.67 in $A(\mathrm{Fe})$ ]. This small scatter is in line with the narrow $[\mathrm{Fe} / \mathrm{H}]$ distribution measured previously by Ramirez et al. (2000) for the same sample. It is much smaller, however, than the spread of more than 1.0 dex found for the metallicity distribution of the older bulge giant population (see, e.g., the histogram in Fig. 8 of Fulbright et al. 2006; Zoccali et al. 2003). The hypothesis that the Galactic center stars are drawn from the bulge metallicity distribution is rejected with $P=\sim 0.001$ using a Kolmogorov-Smirnov (K-S) test. An additional difference between the bulge population and the Galactic center stars sampled here is the luminosity. Frogel \& Whitford (1987) find very few bulge stars with $M_{\text {bol }}$ brighter than -4.2 ; the stars studied here range from $M=-4.5$ to $M=-9.0$. The Galactic center stars studied here were selected to provide a snapshot of current conditions in the Central Molecular Zone; they sample $R_{g}=0.2$ $30 \mathrm{pc}$ and have ages in the range $10 \mathrm{Myr}$ to $1 \mathrm{Gyr}$. It is not yet clear if the narrow Fe-abundance distribution is indicative of a narrow distribution over a larger volume of space or time in the Galactic center, but this can be tested with future abundance studies of the Galactic center and bulge populations.

\subsection{Carbon, Nitrogen, and Oxygen and the Evolutionary State of the Galactic Center Stars}

The CNO elemental trio are all very abundant and are important for studying both chemical and stellar evolution. Three of the program stars were observed across sufficient wavelength intervals such that the most important CNO nuclei could be analyzed. The studied nuclei include ${ }^{12} \mathrm{C},{ }^{14} \mathrm{~N}$, and ${ }^{16} \mathrm{O}$, which are the dominant $\mathrm{CNO}$ species. ${ }^{13} \mathrm{C}$ is not included nor is ${ }^{15} \mathrm{~N}$, although in terms of numbers these are relatively minor species. $\mathrm{As}^{12} \mathrm{C}$ is depleted to levels below ${ }^{14} \mathrm{~N}$ (with ${ }^{14} \mathrm{~N}$ being built up during
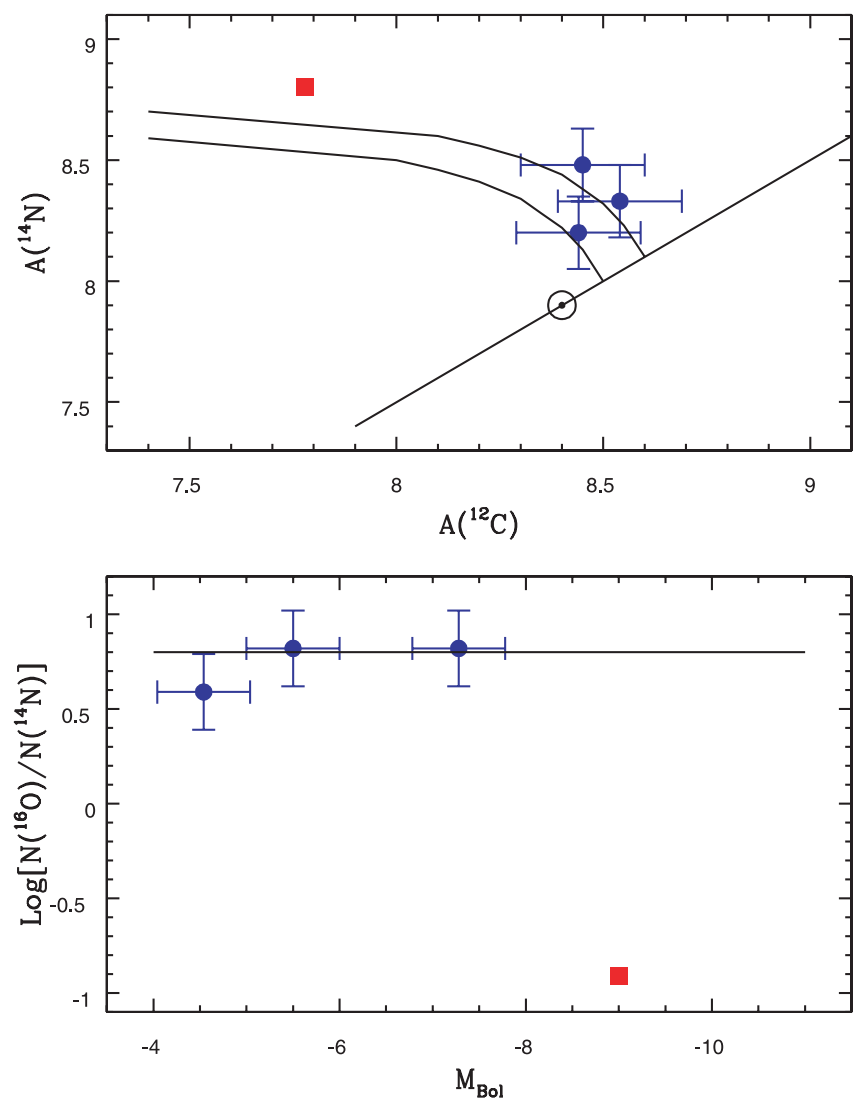

FIG. 6. -Top: Nitrogen $\left[A\left({ }^{14} \mathrm{~N}\right)\right]$ vs. carbon $\left[A\left({ }^{12} \mathrm{C}\right)\right]$ abundances for three Galactic center stars studied here (filled blue circles), as well as IRS 7 (red square; Carr et al. 2000). The solar values are shown, along with a line that defines a solar ${ }^{12} \mathrm{C} /{ }^{14} \mathrm{~N}$ ratio. The solid curves show lines of constant ${ }^{12} \mathrm{C}+{ }^{14} \mathrm{~N}$, or the so-called $\mathrm{CN}$-mixing curves, with initial abundances larger than solar by 0.1 and 0.2 dex, respectively. These curves mimic $\mathrm{CN}$-cycle H-burning in which the sum of ${ }^{12} \mathrm{C},{ }^{14} \mathrm{~N},{ }^{13} \mathrm{C}$, and ${ }^{15} \mathrm{~N}$ is approximately conserved. We do not observe ${ }^{13} \mathrm{C}$ and ${ }^{15} \mathrm{~N}$, but these are minor constituents and would change the curves only slightly. The three Galactic center stars studied here fall within the $\mathrm{CN}$-mixing curves. The very luminous supergiant IRS 7 exhibits much more extreme mixing and the large ${ }^{14} \mathrm{~N}$ abundance may require the addition of $\mathrm{ON}-$ cycle material into the atmosphere. Bottom: ${ }^{16} \mathrm{O} /{ }^{14} \mathrm{~N}$ ratios vs. $M_{\text {bol }}$ for Galactic center stars are shown. The horizontal line denotes the solar ratio as a reference point. The three evolved stars analyzed here show no evidence of significant ${ }^{16} \mathrm{O}$ depletion (from ON-cycle mixing), while the more luminous Galactic center supergiant IRS 7 does show strong evidence for such mixing.

the $\mathrm{CN}$ cycle), the expected ${ }^{12} \mathrm{C} /{ }^{13} \mathrm{C} \mathrm{CN}$-cycle equilibrium ratio of 3.5 will be reached such that $N\left({ }^{13} \mathrm{C}\right)<N\left({ }^{12} \mathrm{C}\right)<N\left({ }^{14} \mathrm{~N}\right)$, so lack of a ${ }^{13} \mathrm{C}$ abundance will not hinder our conclusions about chemical evolution significantly. ${ }^{15} \mathrm{~N}$ is expected to be a very minor constituent, so its contribution to the total abundances is not important for our discussion.

The program stars observed here are almost certainly mixed to some degree, with some fraction of their convective envelope material having been exposed to $\mathrm{CN}$-cycle H-burning, or possibly ON-cycle H-burning. This scenario can be tested using the respective $\mathrm{CNO}$ abundances. If the convective envelopes have been subjected to $\mathrm{CN}$-cycle proton captures, then the total sum of ${ }^{12} \mathrm{C},{ }^{13} \mathrm{C}$, and ${ }^{14} \mathrm{~N}$ will be approximately conserved, and this can be probed by plotting $A\left({ }^{14} \mathrm{~N}\right)$ versus $A\left({ }^{12} \mathrm{C}\right)$, as shown in the top panel of Figure $6 .{ }^{13} \mathrm{C}$ is ignored, as we have no information on it, but ${ }^{13} \mathrm{C}$ will not significantly alter any conclusions obtained from Figure 6. The solid circles with error bars are the ${ }^{12} \mathrm{C}$ and ${ }^{14} \mathrm{~N}$ abundances for Galactic center stars IRS 19, BSD 72, and BSD 124, and the results for IRS 7 by Carr et al. (2000) are represented by the red square. The solar point is plotted with the 
straight line delineating a solar ${ }^{12} \mathrm{C} /{ }^{14} \mathrm{~N}$ ratio scaled in metallicity. The solid curves are defined by constant abundances of ${ }^{12} \mathrm{C}+{ }^{14} \mathrm{~N}$; the two curves show carbon plus nitrogen abundances scaled up from solar by 0.1 and 0.2 dex.

All three stars studied here are well-fit by slightly elevated metallicities (as found from the $\mathrm{Fe}$ abundances) and $\mathrm{CN}$-cycled material in their atmospheres (somewhat depleted ${ }^{12} \mathrm{C}$ and elevated ${ }^{14} \mathrm{~N}$ ). Also shown in the two panels of Figure 6 are the ${ }^{12} \mathrm{C}$ and ${ }^{14} \mathrm{~N}$ abundances for IRS 7 from Carr et al. (2000). IRS 7 is considerably more massive and luminous than IRS 19, BSD 72, or BSD 124 and shows a much lower ${ }^{12} \mathrm{C}$ and much larger ${ }^{14} \mathrm{~N}$ abundance than any of the three lower luminosity stars. The ${ }^{14} \mathrm{~N}$ abundance in IRS 7 is so large that it may indicate that the $\mathrm{CN}$ cycle alone cannot account for such a large amount of nitrogen. Indeed, Carr et al. (2000) find a very low ${ }^{16} \mathrm{O}$ abundance and argue that this is evidence of the presence of ON-cycle material in the atmosphere of IRS 7 (where ${ }^{16} \mathrm{O}$ has been converted to ${ }^{14} \mathrm{~N}$ ). Being much more massive and luminous (it is the brightest star in the Galactic center at $H$ and $K$ ), IRS 7 is presumably more deeply mixed and this suggestion is borne out by the bottom panel of Figure 6, where we show $\log \left[N\left({ }^{16} \mathrm{O}\right) / N\left({ }^{14} \mathrm{~N}\right)\right]$ versus $M_{\text {bol }}$ for the four Galactic center stars.

\section{3. $\alpha$-Element Abundances in the Galactic Center}

Determination of $[\alpha / \mathrm{Fe}]$ in any stellar population and, in particular, in young stars in the Galactic center provides important information on chemical enrichment, gas infall, and gas outflow. One possible scenario is that the relatively young ages (1-100 Myr) of the brightest stars in the Galactic center support the idea that the Galaxy's central bar has driven disk gas into the Galactic center to fuel star formation throughout the Galaxy's history, continuously or in bursts of star formation (Serabyn \& Morris 1996; Morris \& Serabyn 1996; Blum et al. 2003; Figer et al. 2004). In particular, the star formation conditions in the Galactic center are very different from those in the solar neighborhood and are predicted to result in an initial mass function (IMF) weighted toward massive stars (Morris 1993). Furthermore, models of SN enrichment in an environment dominated by massive stars predict a high relative abundance of $\alpha$-elements compared to $\mathrm{Fe},[\alpha / \mathrm{Fe}]$ (e.g., Wheeler et al. 1989).

The results presented in Figure 6 show the presence of $\mathrm{CN}$ mixing in the three evolved stars, but not the very deep mixing that is observed in the much more luminous red supergiant IRS 7. This suggests that oxygen has been effectively untouched by deeper mixing in these three stars. In addition, because the net result of ON-cycle mixing would be to reduce the oxygen abundance, the oxygen results in fact represent a lower limit on the initial oxygen abundance for the original gas from which these stars formed. The average oxygen abundance found for our sample of five stars is $\langle A(\mathrm{O})\rangle=9.04 \pm 0.19$. This can be compared to our adopted solar value of $A(\mathrm{O})=8.70$ (based on [O I] abundances from Asplund et al. [2005], which are $A(\mathrm{O})=8.72$ from one-dimensional models and 8.68 from three-dimensional models). The scatter observed in the sample oxygen abundance distribution can be explained by the uncertainties in the analysis (discussed in $\S 3.4$ ).

The values of $[\mathrm{O} / \mathrm{Fe}]$ in the Galactic center stars can be compared to other samples from the Galaxy. A comparison to the Galactic disk and halo is shown in the top panel of Figure 7. In the five stars studied here for oxygen, it is apparent that the average value of $[\mathrm{O} / \mathrm{Fe}]$ is elevated relative to the disk: $\langle[\mathrm{O} / \mathrm{Fe}]\rangle=$ $+0.22 \pm 0.15$. Both the narrow $[\mathrm{Fe} / \mathrm{H}]$ and elevated $\alpha$-element abundances are reminiscent of the results obtained by Rich \& Origlia (2005) for M giants in the bulge $500 \mathrm{pc}$ from the Galactic center. This behavior of enhanced values of $[\mathrm{O} / \mathrm{Fe}]$ at $[\mathrm{Fe} / \mathrm{H}] \gtrsim 0.0$

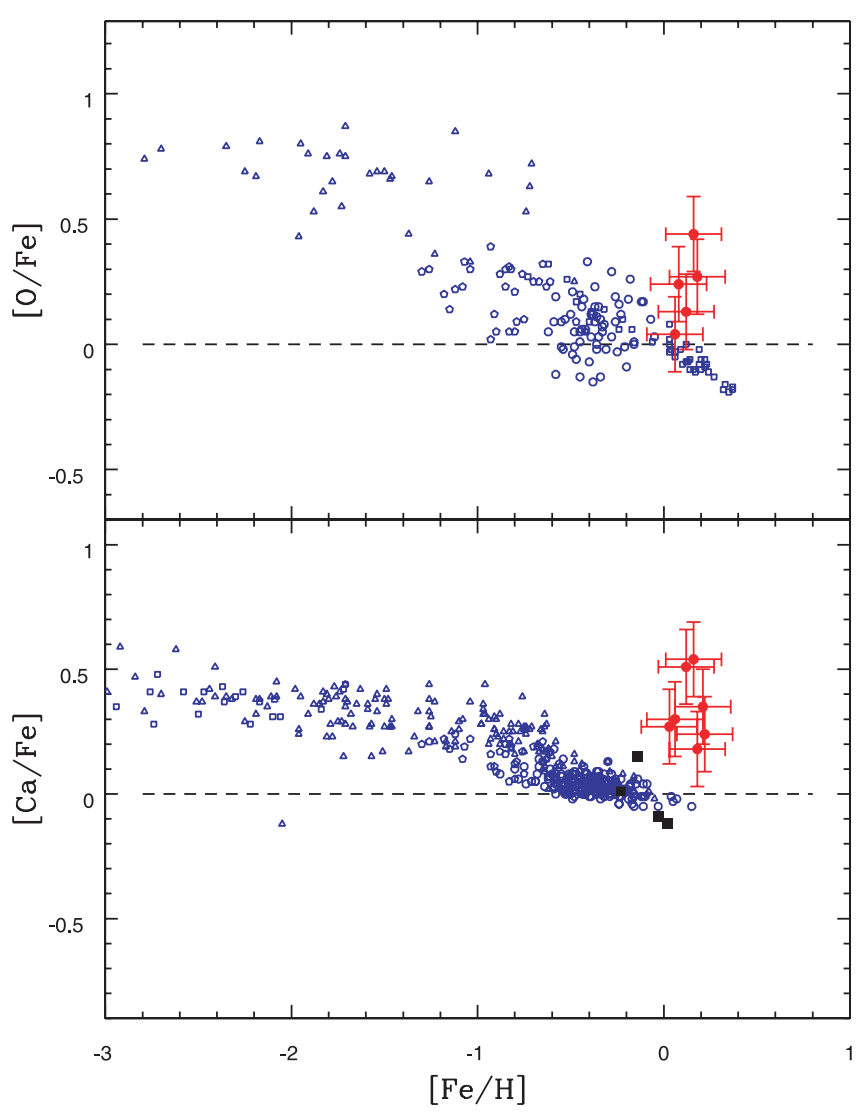

FIG. 7.-Top: Behavior of $[\mathrm{O} / \mathrm{Fe}]$ vs. $[\mathrm{Fe} / \mathrm{H}]$ in different stellar populations. The small open symbols (all in blue) are studies of Galactic disk and halo field stars from Bensby et al. (2004; squares), Reddy et al. (2003; circles), Nissen \& Schuster (1997; pentagons), and Fulbright \& Johnson (2003; triangles). The decrease in $[\mathrm{O} / \mathrm{Fe}]$ as $[\mathrm{Fe} / \mathrm{H}]$ increases is interpreted as increasing contributions from $\mathrm{SNe}$ Ia to $\mathrm{Fe}$ (but no significant O-production from $\mathrm{SNe}$ Ia) as time increases, causing $[\mathrm{O} / \mathrm{Fe}]$ to decline. The Galactic center stars, plotted as the large filled red circles, are displaced significantly to larger values of $[\mathrm{O} / \mathrm{Fe}]$ relative to disk stars at the same $[\mathrm{Fe} / \mathrm{H}]$; this suggests a larger relative contribution from SN II chemical enrichment in the Galactic center population. Bottom: Run of $[\mathrm{Ca} / \mathrm{Fe}]$ values as a function of $[\mathrm{Fe} / \mathrm{H}]$. The trend of $[\mathrm{Ca} / \mathrm{Fe}]$ is similar in shape to that of $[\mathrm{O} / \mathrm{Fe}]$ in that $[\mathrm{Ca} / \mathrm{Fe}]$ is larger in the more metal-poor stars. As calcium, like oxygen, is primarily a product of $\mathrm{SNe} \mathrm{II}$, its behavior should track, to some degree, that of oxygen, as is observed. Again, the Galactic center stars stand out from disk stars in having enhanced values of $[\mathrm{Ca} / \mathrm{Fe}]$. Both $\mathrm{Ca}$ and $\mathrm{O}$ abundances point to the Galactic center stars as being enriched in SN II chemical yields. The filled black squares in this figure are the four bright disk $\mathrm{M}$ giants analyzed for $\mathrm{Ca}$ and $\mathrm{Fe}$ in the same manner as was done for the Galactic center stars. These disk stars follow the same trend in $[\mathrm{Ca} / \mathrm{Fe}]$ vs. $[\mathrm{Fe} / \mathrm{H}]$ as the other disk samples. This result strengthens the conclusion that the Galactic center stars contain elevated values of $[\mathrm{Ca} / \mathrm{Fe}]$.

is also similar to what has been found by a number of different studies of old $\mathrm{K}$ and $\mathrm{M}$ red giants in the Galactic bulge (Cunha \& Smith 2006; Zoccali et al. 2006; Fulbright et al. 2006). The interpretation of the bulge pattern is that the chemical enrichment was rapid and dominated by SNe II to quite high Fe abundances. The population of stars studied here, however, is distinct and very different from the bulge, as the Galactic center stars are all rather young and massive (their estimated masses are presented in Table 1). In addition, the $K$-luminosity function of the bulge only contains stars with $K_{0} \gtrsim 8.0$ (DePoy et al. 1993; Zoccali et al. 2003), while the Galactic center stars observed here have $K_{0}=3.0-7.4$.

This study also includes an additional $\alpha$-element, calcium. The results for calcium, in the form of $[\mathrm{Ca} / \mathrm{Fe}]$ versus $[\mathrm{Fe} / \mathrm{H}]$, are shown in the bottom panel of Figure 7. The small open symbols are results for Galactic field disk and halo from a number of studies 
noted in the figure caption. The large filled symbols are the $[\mathrm{Ca} / \mathrm{Fe}]$ results for the Galactic center stars. Because of the larger number of Galactic center stars with measured $\mathrm{Ca}$ abundances, the elevated values of $[\mathrm{Ca} / \mathrm{Fe}]$ at large metallicity are even more striking than the results for $[\mathrm{O} / \mathrm{Fe}]$. Of further interest are the results for $[\mathrm{Ca} / \mathrm{Fe}]$ in the four disk $\mathrm{M}$ giants analyzed in the same way as the Galactic center; these are plotted as the filled squares. The disk M giants exhibit quite small scatter around the disk trend of $[\mathrm{Ca} / \mathrm{Fe}]$ versus $[\mathrm{Fe} / \mathrm{H}]$. Calcium behaves in a manner similar to oxygen, relative to $\mathrm{Fe}$. The average $[\mathrm{Ca} / \mathrm{Fe}]$ abundance for our Galactic center sample is $\langle[\mathrm{Ca} / \mathrm{Fe}]\rangle=+0.27 \pm$ 0.14 ; here again the abundance spread can be explained by the errors in the analysis. A similar behavior for the two elements oxygen and calcium provides strong evidence that this sample of Galactic center stars are composed of chemical compositions containing larger abundances of the $\alpha$-elements relative to Fe when compared to the Galactic disk at the same metallicity.

The results derived here of elevated $\mathrm{O}$ and $\mathrm{Ca}$ abundances, relative to $\mathrm{Fe}$, are unexpected. It is therefore of interest to carefully evaluate if it is possible that reasonable changes in the adopted stellar parameters could erase the observed $\alpha$-enhancements. The various abundance sensitivities to the different stellar parameters are listed in Table 5. Examination of this table reveals that the only plausible parameter that could be changed in order to drive the elevated values of $[\alpha / \mathrm{Fe}]$ to near solar would be the effective temperature; a change of $\delta T_{\text {eff }} \sim-400 \mathrm{~K}$ would be needed in order to force an average $[\mathrm{Ca} / \mathrm{Fe}] \sim 0.0$ for this sample of stars. Such a systematic shift in the $T_{\text {eff }}$ scale is unlikely. Inspection of Figure 3 shows that such a change would shift $T_{\text {eff }}$ for these Galactic center stars to values of $\sim 2800-3500 \mathrm{~K}$. These lower temperatures would produce strong $\mathrm{H}_{2} \mathrm{O}$ absorption for the coolest stars, which is not observed (Blum et al. 2003), and would put the highest luminosity stars in extreme disagreement with the stellar model tracks (Fig. 4). All this combined seems to indicate that the stellar members of the Central and Quintuplet Clusters analyzed here contain larger than solar ratios of $\mathrm{Ca} / \mathrm{Fe}$ and $\mathrm{O} / \mathrm{Fe}$.

\subsubsection{Comparisons with Other Abundance Measurements} in the Galactic Center

Independent measurements of the abundances of cool, massive stars in the Galactic center are offered by studies of hot, massive stars in the three massive clusters in the central $100 \mathrm{pc}$, and by studies of the gas in the Central Molecular Zone. The massive young stars (formed millions of years ago) and the present-day gas in the central 200 pc both provide snapshots of how far the Galactic center has chemically evolved to date. One important aspect of such comparisons is the fact that very different analysis techniques are used to measure nebular abundances and chemical compositions of lines in the atmospheres of stars.

Najarro et al. (2004) measure the $\mathrm{N}$ abundance from nearinfrared spectra of WNL stars in the Arches Cluster (projected $R_{g}=24 \mathrm{pc}$ ) in the Galactic center. They find best agreement between observations and theory for solar-metallicity stellar evolution models. Najarro (2006) presents preliminary models of the near-infrared spectra of two stars in the Quintuplet Cluster and finds a solar Fe abundance for both. Najarro (2006) also finds initial results that the $\alpha$-elements $\mathrm{Si}$ and $\mathrm{Mg}$ are enhanced by $\sim 0.1-0.3$ dex over solar.

Infrared spectroscopy has probed two $\mathrm{H}$ II regions in the Galactic center, G359.98-0.08 and G0.095+0.012 (ionized by the Arches Cluster), at projected $R_{g}=6.6$ and $24 \mathrm{pc}$, respectively. This reveals enhanced $\alpha$-elements, $[\mathrm{O} / \mathrm{H}]=0.1-0.6$ and $[\mathrm{S} / \mathrm{H}]=0.2-0.4$, and also $[\mathrm{N} / \mathrm{H}]=0.5-0.8$ (Simpson et al. 1995; Afflerbach et al. 1997; Rudolph et al. 2006). Shields \&
Ferland (1994) model the far-infrared spectroscopy of Sgr A West, the $\mathrm{H}$ II region surrounding the Central Cluster. They find a best fit for $[\mathrm{Ar} / \mathrm{H}]=+0.3$ and $[\mathrm{Ne} / \mathrm{H}]=0.0$, and conclude that the overall metallicity is between solar and twice solar. Lutz et al. (1993) measure $[\mathrm{Fe} / \mathrm{H}] \geq-0.1$ in the "mini-cavity" region of Sgr A West, and point out that unless there is absolutely no depletion of Fe onto grains, highly supersolar Fe abundances are required. They suggest that fast shocks from stellar winds have destroyed dust grains and released Fe into the gas.

Recent X-ray measurements of the gas-phase Fe abundance in the Galactic center have been made. Sgr A East has a mean value of $[\mathrm{Fe} / \mathrm{H}]=0.1$ (Sakano et al. 2004) to 0.6 (Maeda et al. 2002). Sgr A East is a young ( $\sim 10^{4}$ yr old $) \mathrm{SN}$ remnant which is physically interacting with the Sgr A West H II region (YusefZadeh \& Morris 1987). Evidence suggests that the Sgr A East SN shock front passed through Sgr A West $\sim 300 \mathrm{yr}$ ago (Maeda et al. 2002). Sakano et al. (2004) measure $[\mathrm{Ca} / \mathrm{H}]=+0.4,[\mathrm{~S} / \mathrm{H}]=$ $+0.3,[\mathrm{Si} / \mathrm{H}]=+0.7$, and $[\mathrm{Ar} / \mathrm{H}]=+0.1$ in Sgr A East. Sgr A East, however, is expected to be self-enriched, and it is under debate what type SN it was. Koyama et al. (2007) find $[\mathrm{Fe} / \mathrm{H}]=$ +0.5 from the absorption column density of Fe nuclei toward the Galactic center; they argue that most of this absorption is from dense clouds local to the Galactic center. The absorption toward the Galactic center, however, is well known to have significant contributions from foreground material (Magnani et al. 2006).

These results on infrared spectroscopy of hot massive stars, infrared spectroscopy of $\mathrm{H}$ II regions, and X-ray spectroscopy of Sgr A East and the diffuse gas in the Galactic center offer an independent assessment of the chemical abundances in the Galactic center. We conclude from the discussion above that there is independent evidence for an enhancement of $\alpha$-elements of $0.2-0.4$ dex in the Galactic center, from $\mathrm{O}$ and $\mathrm{S}$ in the gas, and from $\mathrm{Si}$ and $\mathrm{Mg}$ in hot massive stars. The X-ray Fe abundances are difficult to interpret, but Najarro (2006) find $[\mathrm{Fe} / \mathrm{H}]=0.0$ in hot massive stars, as we do in cool massive stars.

\subsection{Connections with Metallicity Gradients of the Galactic Disk}

It is of interest to compare the abundances of stars located within $60 \mathrm{pc}$ of the Galactic center with the abundances that have been measured further out in the Galactic disk in order to examine the possibility that gas has been driven to the Galactic center, for example, by the presence of the central bar. This comparison can be done via a discussion of the metallicity gradients derived for the more distant parts of the Milky Way and centers around the question of whether these gradients smoothly connect disk abundances to those in the center of the Galaxy. As discussed in Smartt et al. (2001), there seem to be indications of differences between the inner disk and the Galactic center which are inferred from the general lack of $\mathrm{H}$ II regions, molecular gas, and young stars in the region between the central clusters and Galactic radii corresponding to $\sim 3.5 \mathrm{kpc}$.

Stellar abundances as a function of galactocentric distance are illustrated in Figure 8 for the elements oxygen, calcium, and iron. The data are taken from a number of studies as indicated in the figure. The top panel shows $[\mathrm{O} / \mathrm{H}]$ versus $R_{g}$ with disk abundances coming from both OB stars and Cepheids. The Cepheids are much cooler than the OB stars with oxygen abundances in the Cepheids derived from neutral oxygen lines, while in the OB stars $\mathrm{O}$ II lines are analyzed. Despite these different abundance indicators, values of $\mathrm{O} / \mathrm{H}$ for the two groups of stars largely overlap and show a gradual increase of oxygen with a decrease in $R_{g}$ (e.g., Daflon \& Cunha [2004] fit a linear slope of $-0.04 \mathrm{dex} \mathrm{kpc}^{-1}$ 


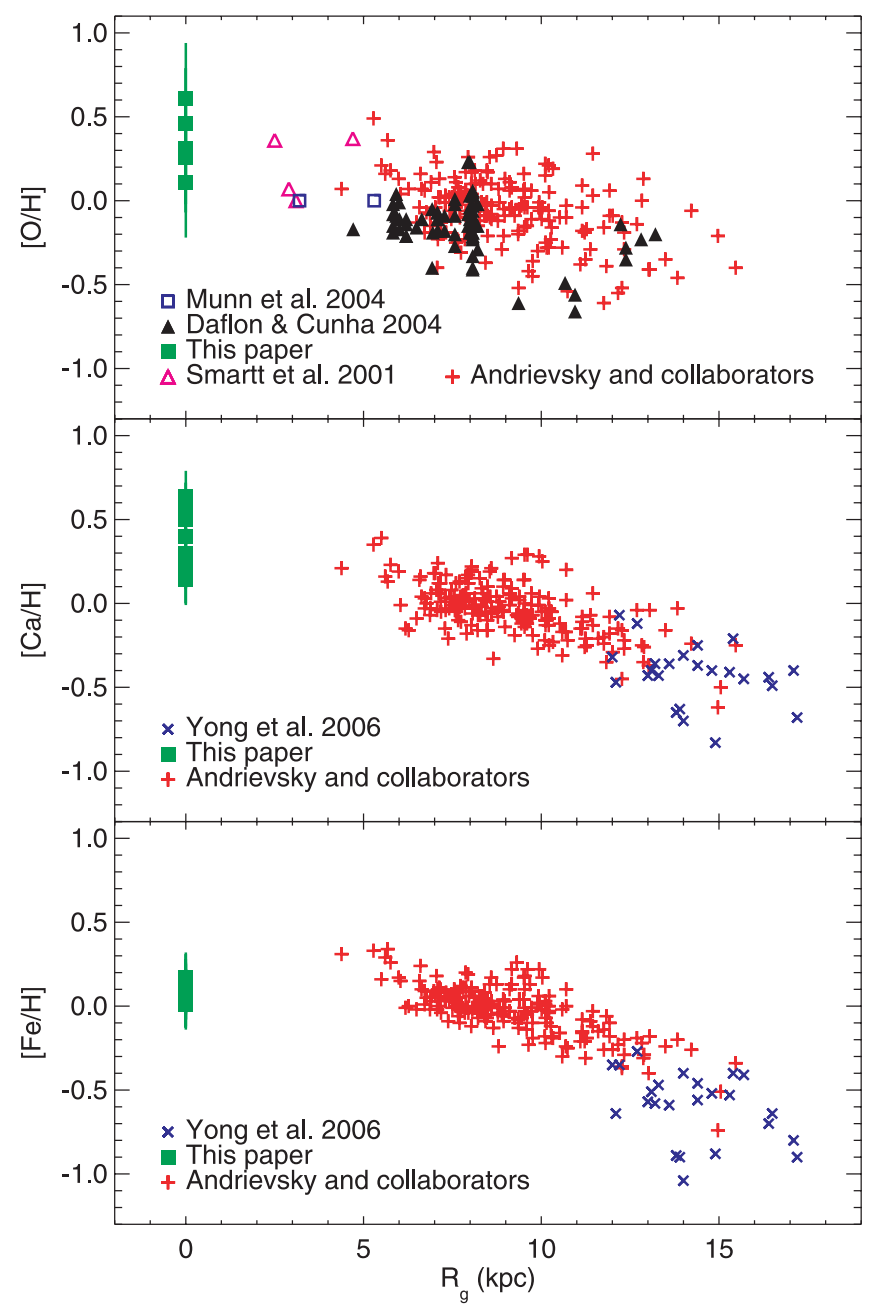

FIG. 8. -Abundance vs. galactocentric distance, $R_{g}$, for the elements $\mathrm{O}$ (top), $\mathrm{Ca}$ (middle), and $\mathrm{Fe}$ (bottom) for disk stars and Galactic center stars. The disk studies include OB stars for [O/H] (Smartt et al. 2001; Daflon \& Cunha 2004; Munn et al. 2004), as well as Cepheids from Andrievsky and collaborators (Andrievsky et al. 2002a, 2002b, 2002c, 2004; Luck et al. 2003, 2006; Kovtyukh et al. 2005). The disk stars for $[\mathrm{Ca} / \mathrm{H}]$ and $[\mathrm{Fe} / \mathrm{H}]$ include Cepheids from Andrievsky and collaborators and from Yong et al. (2006). The disk values for $[\mathrm{O} / \mathrm{H}],[\mathrm{Ca} / \mathrm{H}]$, and $[\mathrm{Fe} / \mathrm{H}]$ show increasing abundances with decreasing $R_{g}$. The Galactic center abundances fall near, or below, a smooth extrapolation of the disk trends.

for their OB star data). Given the scatter in the data points when considering both types of stars, the elevated values of $[\mathrm{O} / \mathrm{H}]$ found here in the Galactic center stars do not seem to be at odds with a gradual and modest increase in the oxygen abundances for $R_{g}$ close to zero.

Although calcium and iron cannot be studied in OB stars, both elements are contained in the more extended list of elements whose abundances can be derived from spectroscopy of Cepheids. The middle panel of Figure 8 shows $[\mathrm{Ca} / \mathrm{H}]$ as a function of galactocentric distance. As with oxygen there is a gradual increase in the $\mathrm{Ca}$ abundance with smaller values of $R_{g}$. In this case, however, there are no Cepheid measurements inside of roughly $5 \mathrm{kpc}$, so there is a large gap in any connection to the Galactic center. Because of the lack of $\mathrm{Ca}$ abundances in the direction of the Galactic center it would be more difficult to argue that the values of $[\mathrm{Ca} / \mathrm{H}]$ found in the stars there represent a smooth extension of the trend observed in the disk.

Concerning iron, Luck et al. (2006), who summarize all of their group's previous studies, find that the overall gradient for the Cepheid sample is $-0.068 \mathrm{dex} \mathrm{kpc}^{-1}$ for galactocentric dis-

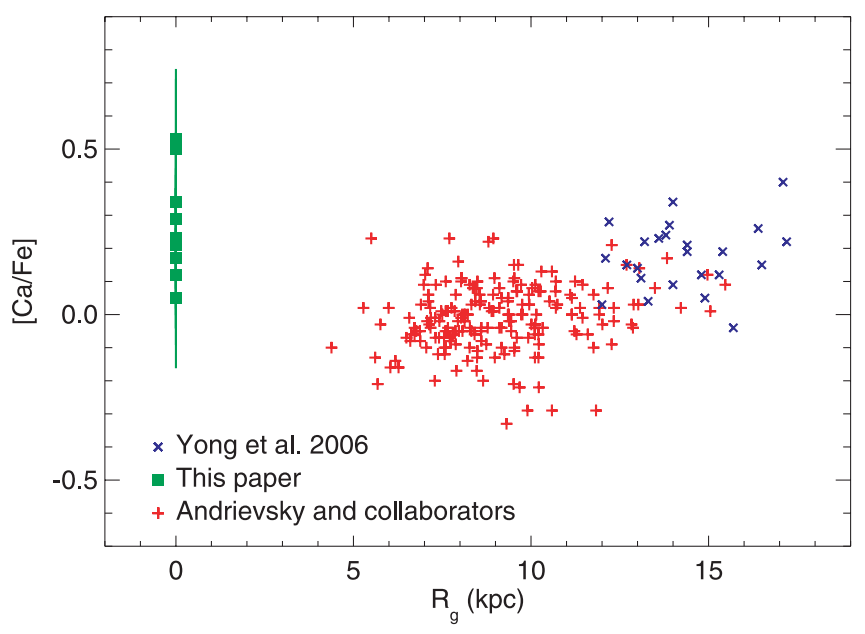

FIG. 9.-Calcium to iron abundance ratios (as $[\mathrm{Ca} / \mathrm{Fe}]$ ) for the Cepheid samples (the same as Fig. 8) and the Galactic center. Values of $[\mathrm{Ca} / \mathrm{Fe}]$ in the disk stars decrease as $R_{g}$ decreases, whereas the Galactic center stars have significantly larger values of $[\mathrm{Ca} / \mathrm{Fe}]$. It would not appear that the Galactic center $\mathrm{Ca}$ and $\mathrm{Fe}$ abundances are represented by smooth extrapolations of the disk trends outside $2.5 \mathrm{kpc}$.

tances ranging between roughly 4 and $16 \mathrm{kpc}$. A simple extrapolation of this iron gradient to $R_{g}=0$ (Galactic center), would yield an iron abundance that is enhanced by +0.6 dex relative to the solar value [or, $A(\mathrm{Fe})=8.1$ ]; this abundance is significantly higher than the average $\mathrm{Fe}$ abundance obtained in this study for the Galactic center stars $[\langle A(\mathrm{Fe})\rangle=7.6]$. This is well illustrated in the bottom panel of Figure 8 . In this case, the gradient in the Fe abundance is measurably steeper than that observed for calcium. In order for the Galactic center stars to have Fe abundances that are representative of the inner disk would require that this trend flattens markedly at small values of $R_{g}$.

The hint that there may be different gradients in the $\mathrm{Ca}$ and Fe abundances can be probed further by examining the ratio of calcium to iron as a function of $R_{g}$; these results are shown in Figure 9. It is clear that there is a general decrease in $[\mathrm{Ca} / \mathrm{Fe}]$ as $R_{g}$ decreases, whereas the Galactic center stars contain elevated values of $[\mathrm{Ca} / \mathrm{Fe}]$. In order for the elemental abundance distributions of the inner disk $\left(R_{g} \sim 3-4 \mathrm{kpc}\right)$ to connect to the Galactic center would require a significant upturn in the trend of $[\mathrm{Ca} / \mathrm{Fe}]$. This could occur as a result of either a rapid increase in $[\mathrm{Ca} / \mathrm{H}]$ or a rapid decrease in $[\mathrm{Fe} / \mathrm{H}]$, or some combination of both. A reexamination of Figure 8 might allow for a turnover in $[\mathrm{Fe} / \mathrm{H}]$ below $R_{g} \sim 4 \mathrm{kpc}$; however, there are no iron abundance data points in this part of the disk. It is perhaps safer to conclude that the significantly enhanced values of $[\mathrm{Ca} / \mathrm{Fe}]$ found in all $10 \mathrm{Ga}-$ lactic center stars studied here do not seem to be consistent with simple extrapolations of disk abundance gradients into the center.

The high value of $[\mathrm{Ca} / \mathrm{Fe}]$ observed in the Galactic center stars might argue against gas from the disk, which has roughly a solar value of $[\mathrm{Ca} / \mathrm{Fe}]$, reaching the Galactic center. Similarly, the narrow $[\mathrm{Fe} / \mathrm{H}]$ distribution observed in the Galactic center stars might argue against gas from the bulge, which has a broad $[\mathrm{Fe} / \mathrm{H}]$ distribution, being a major contributor to the present-day abundances in the Galactic center. Disk gas driven into the Galactic center by the bar, however, and infall of gas from stellar mass loss in the bulge are both believed to fuel sustained star formation in the Galactic center (Serabyn \& Morris 1996; Morris \& Serabyn 1996; Stark et al. 2004). Furthermore, we observe enrichment of the Galactic center gas in the present day, in the locally high abundances observed for the SN remnant Sgr A East (Sakano et al. 
2004). The unique abundance pattern in Galactic center stars may reflect the variety of contributors to the chemical evolution of this region.

\subsection{Implications for Chemical Evolution in the Galactic Center}

Chemical evolution models for the specific case of the central $100 \mathrm{pc}$ of the Galactic center, which take into account its extended star formation history as well as its various sources of gas infall and possible mechanisms for gas outflow, are not available in the literature. Such detailed modeling would be extremely welcome for the interpretation of existing and future Galactic center abundance results. In lieu of such detailed models, our abundance results can be considered in a more simplified discussion of chemical evolution.

The evidence presented here of large values of $[\mathrm{O} / \mathrm{Fe}]$ and $[\mathrm{Ca} / \mathrm{Fe}]$ in Galactic center stars points to the dominant role played by SN II chemical enrichment in the gas from which these particular stars formed. This result is not expected from simple star formation history (SFH) and chemical enrichment models. For example, Figer et al. (2004) found that the Galactic center luminosity function could be fit by a continuous star formation history (see Blum et al. 2003 for a more general treatment which does not lead to a continuous SFH). In a continuous SFH (or a SFH with repeated bursts of star formation), SNe Ia will begin to contribute, after $\sim 1$ Gyr, large amounts of Fe and drive the $[\mathrm{O} / \mathrm{Fe}]$ and $[\mathrm{Ca} / \mathrm{Fe}]$ values down toward ones more solar-like in the younger stars; such solar-like ratios are not observed here.

There are solutions that would reconcile the picture of continuous star formation with the enhanced values of $[\alpha / \mathrm{Fe}]$ observed here in young Galactic center stars. One could invoke either an IMF that is weighted toward more massive stars or appeal to the stars' youth and their restricted location in space. In a top-heavy IMF, the larger fraction of massive stars would overproduce both oxygen and calcium when compared to disk values. In this case, the Fe produced by the lower mass SN Ia systems would not be enough to yield values of $[\alpha / \mathrm{Fe}]=0$ in the gas from which the young stars formed. A second scenario would argue that the star formation process that has produced the massive clusters near the Galactic center could have self-polluted their local region on short timescales via SNe II within the clusters themselves. Continuing star formation within the cluster environment would then occur in gas enriched in calcium and oxygen. This scenario would mean that the elevated values of $[\mathrm{O} / \mathrm{Fe}]$ and $[\mathrm{Ca} / \mathrm{Fe}]$ are peculiar to the time and place occupied by the massive clusters. Olive \& Schramm (1982) suggested that such an effect may occur in large regions of active star formation. A third possibility might be that the gas to drive star formation arrived at the Galactic center from an outside reservoir. A relatively nearby source of $\alpha$-enhanced high-metallicity material exists in the old bulge population. Red giant members of the bulge have been evolving and losing mass over many Gyr via low-velocity red giant winds. Mass loss from bulge red giants has been suggested as a fuel for sustained star formation in the Galactic center (Morris \& Serabyn 1996). The average abundances from a mixture of bulge red giant winds (averaged over the metallicity distribution of the bulge) closely resembles the $\mathrm{O}, \mathrm{Ca}$, and $\mathrm{Fe}$ abundance patterns found for the Galactic center stars. An analysis of a more extended sample of Galactic center stars could potentially test the scenarios discussed above.

\section{CONCLUSIONS}

The 10 cool, luminous, evolved Galactic center stars analyzed here present nearly uniform iron abundances that are slightly larger than solar, with a mean and standard deviation of $A(\mathrm{Fe})=$ $7.59 \pm 0.06$ (or $[\mathrm{Fe} / \mathrm{H}]=+0.14$ ). This value for $\mathrm{Fe}$ confirms the near-solar, or slightly elevated values reported previously in Galactic center stars by Ramirez et al. (2000) and Carr et al. (2000). The derived small scatter in the Fe abundances is within the uncertainty of an individual star's abundance; thus, a single-valued iron abundance typifies the particular Galactic center population sampled by these 10 stars. Similarly, the oxygen and calcium abundances for the studied sample can also be represented by single abundance values within the expected uncertainties in the analysis.

Analysis of the specific CNO isotopes ${ }^{12} \mathrm{C},{ }^{14} \mathrm{~N}$, and ${ }^{16} \mathrm{O}$ reveals the presence of $\mathrm{CN}$-cycle material having been mixed into the atmospheres of these evolved stars; that is, ${ }^{14} \mathrm{~N}$ is significantly enhanced, accompanied by a slightly lower ${ }^{12} \mathrm{C}$ abundance, such that the sum of ${ }^{12} \mathrm{C}$ and ${ }^{14} \mathrm{~N}$ nuclei is roughly constant, as expected for $\mathrm{CN}$-cycle mixing. The three stars studied for $\mathrm{CNO}$ fall along so-called $\mathrm{CN}$-mixing lines in a ${ }^{14} \mathrm{~N}$ versus ${ }^{12} \mathrm{C}$ diagram for an initial ${ }^{12} \mathrm{C} /{ }^{14} \mathrm{~N}$ value that is about solar.

Perhaps the most significant result of this study is the $\alpha$-enhanced elemental abundances found for the Galactic center stars. The $[\mathrm{O} / \mathrm{Fe}]$ and $[\mathrm{Ca} / \mathrm{Fe}]$ values are on average +0.2 to +0.3 dex higher than typical disk values at similar Fe abundances. These abundance patterns are consistent with a larger fraction of $\mathrm{SNe}$ II relative to $\mathrm{SNe}$ Ia when compared to the disk. A reason for this might be an IMF for the Galactic center weighted toward more massive stars or recent local SN II chemical enrichment within the central $50 \mathrm{pc}$ of the Galaxy, or a mixture of bulge red-giant winds feeding the Galactic center environment.

K. C. thanks Ken Freeman for discussions. K. S. thanks Marc Pinsonneault and Rick Pogge for discussions. These findings are based on observations obtained at the Gemini Observatory, which is operated by the Association of Universities for Research in Astronomy, Inc., under a cooperative agreement with the NSF on behalf of the Gemini partnership: the National Science Foundation (United States), the Particle Physics and Astronomy Research Council (United Kingdom), the National Research Council (Canada), CONICYT (Chile), the Australian Research Council (Australia), CNPq (Brazil), and CONICRT (Argentina). This paper uses data obtained with the Phoenix infrared spectrograph, developed and operated by the National Optical Astronomy Observatory. These findings are also based on observations at the Infrared Telescope Facility, which is operated by the University of Hawaii under cooperative agreement NCC 5-538 with the National Aeronautics and Space Administration, Science Mission Directorate, Planetary Astronomy Program. This work is also supported in part by the National Science Foundation through AST 02-06331 (K. S., S. V. R.) and AST 06-46790 (K. C., V. V. S.), and NASA through JPL Agreement 1265550 (K. S.) and NAG 059213 (K. C., V. V. S.).

\section{REFERENCES}

Afflerbach, A., Churchwell, E., \& Werner, M. W. 1997, ApJ, 478, 190

Andrievsky, S. M., Bersier, D., Kovtyukh, V. V., Luck, R. E., Maciel, W. J., Lepine, J. R. D., \& Beletsky, Yu. V. 2002a, A\&A, 384, 140

Andrievsky, S. M., Kovtyukh, V. V., Luck, R. E., Lepine, J. R. D.,Maciel,W. J., \& Beletsky, Yu. 2002b, A\&A, 392, 491
Andrievsky, S. M., Luck, R. E., Martin, P., \& Lepine, J. R. D. 2004, A\&A, 413, 159 Andrievsky, S. M., et al. 2002c, A\&A, 381, 32

Asplund, M., Grevesse, N., \& Sauval, A. J. 2005, in ASP Conf. Ser. 336, Cosmic Abundances as Records of Stellar Evolution and Nucleosynthesis, ed. F. N. Bash \& T. G. Barnes (San Francisco: ASP), 25 
Bensby, T., Feltzing, S., \& Lundström, I. 2004, A\&A, 415, 155

Bessel, M. S., Castelli, F., \& Plez, B. 1998, A\&A, 333, 231

Blommaert, J. A. D. L., van der Veen, W. E. C. J., van Langevelde, H. J., Habing, H. J., \& Sjouwerman, L. O. 1998, A\&A, 329, 991

Blum, R. D., Ramirez, S. V., Sellgren, K., \& Olsen, K. 2003, ApJ, 597, 323

Blum, R. D., Schaerer, D., Pasquali, A., Heydari-Malayeri, M., Conti, P. S., \& Schmutz, W. 2001, AJ, 122, 1875

Blum, R. D., Sellgren, K., \& DePoy, D. L. 1996a, AJ, 112, 1988 1996b, ApJ, 470, 864

Carr, J. S., Sellgren, K., \& Balachandran, S. C. 2000, ApJ, 530, 307

Cunha, K., \& Smith, V. V. 2006, ApJ, 651, 491

Daflon, S., \& Cunha, K. 2004, ApJ, 617, 1115

DePoy, D. L., Terndrup, D. M., Frogel, J. A., Atwood, B., \& Blum, R. 1993, AJ, 105, 2121

Figer, D. F., Kim, S. S., Morris, M., Serabyn, E., Rich, R. M., \& McLean, I. S. 1999b, ApJ, 525, 750

Figer, D. F., McLean, I. S., \& Morris, M. 1999a, ApJ, 514, 202

Figer, D. F., Najarro, F., Morris, M., McLean, I. S., Geballe, T. R., Ghez, A. M., \& Langer, N. 1998, ApJ, 506, 384

Figer, D. F., Rich, R. M., Kim, S. S., Morris, M., \& Serabyn, E. 2004, ApJ, 601, 319

Frogel, J. A., \& Whitford, A. E. 1987, ApJ, 320, 199

Fulbright, J. P., \& Johnson, J. A. 2003, ApJ, 595, 1154

Fulbright, J., McWilliam, A., \& Rich, M. R. 2006, ApJ, 636, 821

Genzel, R., Pichon, C., Eckart, A., Gerhard, O. E., \& Ott, T. 2000, MNRAS, 317,348

Ghez, A. M., Klein, B. C., Morris, M., \& Becklin, E. E. 1998, ApJ, 509, 678

Ghez, A. M., Salim, S.,Hornstein, S. D., Tanner, A., Lu, J. R., Morris, M., Becklin, E. E., \& Duchene, G. 2005, ApJ, 620, 744

Goorvitch, D. 1994, ApJS, 95, 535

Gray, D. F. 1992, The Observation and Analysis of Stellar Photospheres (Cambridge: Cambridge Univ. Press)

Hinkle, K. H., et al. 2003, Proc. SPIE, 4834, 353

Kleinmann, S. G., \& Hall, D. N. B. 1986, ApJS, 62, 501

Kovtyukh, V. V., Wallerstein, G., \& Andrievsky, S. M. 2005, PASP, 117, 1173

Koyama, K., et al. 2007, PASJ, 59, 245

Krabbe, A., et al. 1995, ApJ, 447, L95

Kuijken, K., \& Rich, R. M. 2002, AJ, 124, 2054

Lambert, D. L., Brown, J. A., Hinkle, K. H., \& Johnson, H. R. 1984, ApJ, 284, 223

Levesque, E. M., Massey, P., Olsen, K. A. G., Plez, B., Josselin, E., Maeder, A., \& Meynet, G. 2005, ApJ, 628, 973

Luck, R. E., Gieren, W. P., Andrievsky, S. M., Kovtyukh, V. V., Fouqué, P., Pont, F., \& Kienzle, F. 2003, A\&A, 401, 939

Luck, R. E., Kovtyukh, V. V., \& Andrievsky, S. M. 2006, AJ, 132, 902

Lutz, D., Krabbe, A., \& Genzel, R. 1993, ApJ, 418, 244

Maeda, Y., et al. 2002, ApJ, 570, 671

Magnani, L., Zelenik, S., Dame, T. M., \& Engebreth, B. 2006, ApJ, 636, 267

Moneti, A., Glass, I. S., \& Moorwood, A. F. M. 1994, MNRAS, 268, 194

Morris, M. 1993, ApJ, 408, 496

Morris, M., \& Serabyn, E. 1996, ARA\&A, 34, 645

Munn, K. E., Dufton, P. L., Smartt, S. J., \& Hambly, N. C. 2004, A\&A, 419, 713
Najarro, F. 2006, J. Phys. Conf. Ser., 54, 224

Najarro, F., Figer, D. F., Hillier, D. J., \& Kudritzki, R. P. 2004, ApJ, 611, L105

Najarro, F., Hillier, D. J., Kudritzki, R. P., Krabbe, A., Genzel, R., Lutz, D., Drapatz, S., \& Geballe, T. R. 1994, A\&A, 285, 573

Nissen, P. E., \& Schuster, W. J. 1997, A\&A, 326, 751

Olive, K. A., \& Schramm, D. N. 1982, ApJ, 257, 276

Plez, B. 1992, A\&AS, 94, 527

Plez, B., Brett, J. M., \& Nordlund, A. 1992, A\&A, 256, 551

Ramirez, S. V., Carr, J. S., Balachandran, S. C., Blum, R., Terndrup, D. A., \& Steed, A. 2000, ApJ, 537, 205

Rich, R. M., \& Origlia, L. 2005, ApJ, 634, 1293

Reddy, Bacham, E., Tomkin, J., Lambert, D. L., \& Allende Prieto, C. 2003, MNRAS, 340, 304

Reid, M. J. 1993, ARA\&A, 31, 345

Rudolph, A. L., Fich, M., Bell, G. R., Norsen, T., Simpson, J. P., Haas, M. R., \& Erickson, E. F. 2006, ApJS, 162, 346

Sakano, M., Warwick, R. S., Decourchelle, A., \& Predehl, P. 2004, MNRAS, 350,129

Salasnich, B., Girardi, L., Weiss, A., \& Chiosi, C. 2000, A\&A, 361, 1023

Schödel, R., Ott, T., Genzel, R., Eckart, A., Mouawad, N., \& Alexander, T. 2003, ApJ, 596, 1015

Sellgren, K., Hall, D. N. B., Kleinmann, S. G., \& Scoville, N. Z. 1987, ApJ, 317,881

Serabyn, E., Lacy, J. H., \& Achtermann, J. M. 1991, ApJ, 378, 557

Serabyn, E., \& Morris, M. 1996, Nature, 382, 602

Shields, J. C., \& Ferland, G. J. 1994, ApJ, 430, 236

Simpson, J. P., Colgan, S. W. J., Rubin, R. H., Erickson, E. F., \& Haas, M. R. 1995, ApJ, 444, 721

Smartt, S. J., Venn, K. A., Dufton, P. L., Lennon, D. J., Rolleston, W. R. J., \& Keenan, F. P. 2001, A\&A, 367, 86

Smith, V. V., \& Lambert, D. L. 1985, ApJ, 294, 326 1986, ApJ, 311, 843 1990, ApJS, 72, 387

Smith, V. V., Suntzeff, N. B., Cunha, K., Gallino, R., Busso, M., Lambert, D. L., \& Straniero, O. 2000, AJ, 119, 1239

Smith, V. V., et al. 2002, AJ, 124, 3241

Sneden, C. 1973, ApJ, 184, 839

Stark, A. A., Martin, C. L., Walsh, W. M., Xiao, K., Lane, A. P., \& Walker, C. K. 2004, ApJ, 614, L41

Tanner, A., Ghez, A. M., Morris, M. R., \& Christou, J. C. 2005, ApJ, 624, 742 Tokunaga, A. T., Toomey, D. W., Carr, J. S., Hall, D. N. B., \& Epps, H. W. 1990, Proc. SPIE, 1235, 131

Wheeler, J. C. Sneden, C., \& Truran, J. W. 1989, ARA\&A, 27, 279

Yamashita, Y. 1971, Publ. Dominion Astrophys. Obs., 13, 47

Yong, D., Aoki, W., Lambert, D. L., \& Paulson, D. B. 2006, ApJ, 639, 918

Yusef-Zadeh, F., \& Melia, F. 1992, ApJ, 385 L41

Yusef-Zadeh, F., \& Morris, M. 1987, ApJ, 320, 545 . 1991, ApJ, 371, L59

Zoccali, M., et al. 2003, 399, 931

. 2006, A\&A, 457, L1 